hep-th/9509066, RU-95-48, IASSNS-HEP-95/70

\title{
LECTURES ON SUPERSYMMETRIC GAUGE THEORIES AND ELECTRIC-MAGNETIC DUALITY*
}

\author{
K. INTRILIGATOR ${ }^{1,2}$ and N. SEIBERG ${ }^{1,2}$ \\ ${ }^{1}$ Department of Physics, Rutgers University \\ Piscataway, NJ 08855-0849, USA \\ ${ }^{2}$ Institute for Advanced Study \\ Princeton, NJ 08540, USA
}

\begin{abstract}
We review some of the recent work on the dynamics of four dimensional, supersymmetric gauge theories. The kinematics are largely determined by holomorphy and the dynamics are governed by duality. The results shed light on the phases of gauge theories. Some results and interpretations which have not been published before are also included.
\end{abstract}

\section{Introduction}

Recently, it has become clear that certain aspects of four dimensional supersymmetric field theories can be analyzed exactly, providing a laboratory for the analysis of the dynamics of gauge theories (for a recent elementary presentation and a list of references see [1]). For example, the phases of gauge theories and the mechanisms for phase transitions can be explored in this context. The dynamical mechanisms explored are standard to gauge theories and thus, at least at a qualitative level, the insights obtained are expected to also be applicable for non-supersymmetric theories. We summarize some of the recent ideas. The discussion is not in historical order and other examples appear in the literature.

\subsection{Phases of gauge theories}

The phases of gauge theories can be characterized by the potential $V(R)$ between electric test charges separated by a large distance $R$. Up to a non-universal, additive

\footnotetext{
* To appear in the Proc. of Trieste '95 spring school, TASI '95, Trieste '95 summer school, and Cargese '95 summer school.
} 
constant, the potential is conjectured to behave as

$$
\begin{aligned}
\text { Coulomb } & V(R) & \sim \frac{1}{R} \\
\text { free electric } & V(R) & \sim \frac{1}{R \log (R \Lambda)} \\
\text { free magnetic } & V(R) & \sim \frac{\log (R \Lambda)}{R} \\
\text { Higgs } & V(R) & \sim \text { constant } \\
\text { confining } & V(R) & \sim \sigma R .
\end{aligned}
$$

The first three phases have massless gauge fields and potentials of the form $V(R) \sim e^{2}(R) / R$. In the Coulomb phase, the electric charge $e^{2}(R) \sim$ constant. In the free electric phase, massless electrically charged fields renormalize the charge to zero at long distances as $e^{-2}(R) \sim \log (R \Lambda)$. Similar behavior occurs when the long distance theory is a non-Abelian theory which is not asymptotically free. The free magnetic phase occurs when there are massless magnetic monopoles, which renormalize the electric coupling constant to infinity at large distance with a conjectured behavior $e^{2}(R) \sim \log (R \Lambda)$. In the Higgs phase, the condensate of an electrically charged field gives a mass gap to the gauge fields by the Anderson-Higgs mechanism and screens electric charges, leading to a potential which, up to the additive non-universal constant, has an exponential Yukawa decay to zero at long distances. In the confining phase, there is a mass gap with electric flux confined into a thin tube, leading to the linear potential with string tension $\sigma$.

All of the above phases can be non-Abelian as well as Abelian. In particular, in addition to the familiar Abelian Coulomb phase, there are theories which have a non-Abelian Coulomb phase with massless interacting quarks and gluons exhibiting the above Coulomb potential. This phase occurs when there is a non-trivial, infrared fixed point of the renormalization group. These are thus non-trivial, interacting four dimensional conformal field theories.

We can also consider the behavior of the potential $V(R)$ for magnetic test charges separated by a large distance $R$. Up to an additive, non-universal constant, the potential behaves as

$$
\begin{aligned}
\text { Coulomb } & V(R) & \sim \frac{1}{R} \\
\text { free electric } & V(R) & \sim \frac{\log (R \Lambda)}{R} \\
\text { free magnetic } & V(R) & \sim \frac{1}{R \log (R \Lambda)} \\
\text { Higgs } & V(R) & \sim \rho R \\
\text { confining } & V(R) & \sim \text { constant. }
\end{aligned}
$$


The behavior in the first three phases can be written as $V(R)=g^{2}(R) / R$ where the effective magnetic charge $g^{2}(R)$ is related to the effective electric charge appearing in (1.1) by the Dirac condition, $e(R) g(R) \sim 1$. The linear potential in the Higgs phase reflects the string tension in the Meissner effect.

The above behavior is modified when there are matter fields in the fundamental representation of the gauge group because virtual pairs can be popped from the vacuum and completely screen the sources. Indeed, in this situation there is no invariant distinction between the Higgs and the confining phases [2]. In particular, there is no phase with a potential behaving as the "confining" potential in (1.1) at large distances - the flux tube can break. For large expectation values of the fields, a Higgs description is most natural while, for small expectation values, it is more natural to interpret the theory as "confining." Because there is really no distinction, it is possible to smoothly interpolate from one interpretation to the other.

Note that under electric-magnetic duality, which exchanges electrically charged fields with magnetically charged fields, the behavior in the free electric phase is exchanged with that of the free magnetic phase. Mandelstam and 'tHooft suggested that, similarly, the Higgs and confining phases are exchanged by duality. Confinement can then be understood as the dual Meissner effect associated with a condensate of monopoles. As we will review, in supersymmetric theories it is possible to show that this picture is indeed correct.

Dualizing a theory in the Coulomb phase, we remain in the same phase (the behavior of the potential is unchanged). For an Abelian Coulomb phase with free massless photons, this follows from a standard duality transformation. What is not obvious is that this is also the case in a non-Abelian Coulomb phase. This was first suggested by Montonen and Olive [3]. The simplest version of their proposal is true only in $N=4$ supersymmetric field theories $[4$ and in finite $N=2$ supersymmetric theories [5,66,7]. The extension of these ideas to asymptotically free $N=1$ theories first appeared in [8] and will be reviewed here.

\subsection{Super Yang-Mills theories}

We briefly review standard lore concerning $N=1$ supersymmetric Yang-Mills theories - i.e. pure super glue with no matter. The lore presented here can be proven via the analysis in the following sections, by adding vector-like matter and then integrating it out.

We consider a theory based on a simple group $G$; the generalization to semisimple Yang-Mills theories is obvious. The theory consists of the $G$ vector bosons and gauginos $\lambda_{\alpha}$ in the adjoint of $G$. There is a classical $U(1)_{R}$ symmetry, gaugino number, which is broken to a discrete $Z_{2 h}$ subgroup by instantons, $\left\langle(\lambda \lambda)^{h}\right\rangle=$ const. $\Lambda^{3 h}$, where $h=C_{2}(A)$ is the Casimir in the adjoint normalized so that, for example, $h=N_{c}$ for $S U\left(N_{c}\right)$. The lore is that this theory confines, gets a mass 
gap, and that there are $h$ vacua associated with the spontaneous breaking of the $Z_{2 h}$ symmetry to $Z_{2}$ by gaugino condensation, $\langle\lambda \lambda\rangle=$ const. $\Lambda^{3}$. These vacua each contribute $(-1)^{F}=1$ and thus the Witten index [9] is $\operatorname{Tr}(-1)^{F}=C_{2}(A)$.

\subsection{Outline}

In sect. 2, we discuss general techniques for analyzing supersymmetric theories. In sect. 3, we discuss the classical moduli spaces of degenerate vacua which supersymmetric gauge theories with matter often have. In particular, we discuss the classical vacuum degeneracy for $N=1$ supersymmetric QCD. In sect. 4, we discuss supersymmetric QCD for $N_{f} \leq N_{c}+1$ massless flavors of quarks in the fundamental representation of $S U\left(N_{c}\right)$. In sect. 5 , we discuss $N_{f}>N_{c}+1$ and duality. Sect. 6 is a brief discussion of the phases and duality of supersymmetric $S O\left(N_{c}\right)$ with matter fields in the $N_{c}$ dimensional representation. In sect. 7 , we discuss general aspects of supersymmetric theories which have a low energy Abelian Coulomb phase. In sect. 8 , we consider the examples of $S U(2)$ with $N_{f}=1$ and $N_{f}=2$ adjoints, which have electric-magnetic-dyonic triality.

\section{Holomorphy and symmetries}

\subsection{General considerations}

The basic approach will be to consider the low energy effective action for the light fields, integrating out degrees of freedom above some scale. Assuming that we are working above the scale of possible supersymmetry breaking, the effective action will have a linearly realized supersymmetry which can be made manifest by working in terms of superfields. The light matter fields can be combined into chiral superfields $X_{r}=\phi_{r}+\theta_{\alpha} \psi_{r}^{\alpha}+\ldots$, where the $\phi_{r}$ are scalars and the $\psi_{r}^{\alpha}$ are Weyl fermions. In addition, there are the conjugate anti-chiral superfields $X_{r}^{\dagger}=\phi_{r}^{\dagger}+\bar{\theta}_{\dot{\alpha}} \psi_{r}^{\dagger \dot{\alpha}}+\ldots$. Similarly, light gauge fields combine into supermultiplets involving a gauge boson $A_{\mu}$ and gauginos $\lambda_{\alpha}$ and $\lambda_{\dot{\alpha}}^{\dagger}$.

We will focus on a particular contribution to the effective Lagrangian - the superpotential term

$$
\int d^{2} \theta W_{\mathrm{eff}}\left(X_{r}, g_{I}, \Lambda\right)
$$

where $X_{r}$ are the light fields, the $g_{I}$ are various coupling constants, and $\Lambda$ is the

dimensional transmutation scale associated with the gauge dynamics, $-\frac{8 \pi^{2}}{g^{2}(\mu)} \sim$ $\log \Lambda / \mu$. Upon doing the $\theta$ integral, the superpotential yields a potential for the scalars and a Yukawa type interaction with the scalars and the fermions.

The key fact is that supersymmetry requires $W_{\text {eff }}$ to be holomorphic in the chiral superfields $X_{r}$, independent of the $X_{r}^{\dagger}$. 
We will think of all the coupling constants $g_{I}$ in the tree level superpotential $W_{\text {tree }}$ and the scale $\Lambda$ as background fields [10]. Then, the quantum, effective superpotential, $W_{\text {eff }}\left(X_{r}, g_{I}, \Lambda\right)$ is constrained by:

1. Symmetries and selection rules: By assigning transformations laws both to the fields and to the coupling constants, the theory has a large symmetry. The effective Lagrangian should be invariant under it.

2. Holomorphy: $W_{\text {eff }}$ is independent of $g_{I}^{\dagger}$ [10]. This is the key property. Just as the superpotential is holomorphic in the fields, it is also holomorphic in the coupling constants (the background fields). This is unlike the effective Lagrangian in nonsupersymmetric theories, which is not subject to any holomorphy restrictions. This use of holomorphy extends considerations of [11,12, 13]. It is similar in spirit to the proofs of non-renormalization in sigma model perturbation theory [14] and in semi-classical perturbation theory [15] in string theory.

3. Various limits: $W_{\text {eff }}$ can be analyzed approximately at weak coupling. The singularities have physical meaning and can be controlled.

Often these conditions completely determine $W_{\text {eff }}$. The point is that a holomorphic function (more precisely, a section) is determined by its asymptotic behavior and singularities. The results can be highly non-trivial, revealing interesting nonperturbative dynamics.

When there is a Coulomb phase, the kinetic terms for the gauge fields are also constrained by the above considerations. The relevant term in the effective Lagrangian is

$$
\int d^{2} \theta \operatorname{Im}\left[\tau_{\mathrm{eff}}\left(X_{r}, g_{I}, \Lambda\right) W_{\alpha}^{2}\right]
$$

$W_{\alpha}^{2}$ gives the supersymmetric completion of $F^{2}+i F \widetilde{F}$ so

$$
\tau_{\mathrm{eff}} \sim \frac{\theta_{\mathrm{eff}}}{2 \pi}+\frac{4 \pi i}{g_{\mathrm{eff}}^{2}}
$$

is the effective gauge coupling constant. $\tau_{\text {eff }}\left(X_{r}, g_{I}, \Lambda\right)$ is holomorphic in its arguments and can often be exactly determined.

\subsection{Example: Wess-Zumino Model}

In order to demonstrate the above rules, we consider the simplest Wess-Zumino model and rederive the known non-renormalization theorem. Consider the theory based on the tree level superpotential

$$
W_{\text {tree }}=m \phi^{2}+g \phi^{3} .
$$


We will make use of two $U(1)$ symmetries. The charges of the field $\phi$ and the coupling constants $m$ and $g$ are

$\begin{array}{cccc} & U(1) & \times & U(1)_{R} \\ \phi & 1 & & 1 \\ m & -2 & & 0 \\ g & -3 & & -1\end{array}$

where $U(1)_{R}$ is an $R$ symmetry under which $d^{2} \theta$ has charge -2 and thus, in order for (2.1) to be invariant, the superpotential has charge 2. Note that non-zero values for $m$ and $g$ explicitly break both $U(1)$ symmetries. However, they still lead to selection rules.

The symmetries and holomorphy of the effective superpotential restrict it to be of the form

$$
W_{\mathrm{eff}}=m \phi^{2} f\left(\frac{g \phi}{m}\right) .
$$

Consider the limit $g \rightarrow 0$ and $m \rightarrow 0$ with arbitrary $g / m$. We must have $W_{\text {eff }} \rightarrow$ $W_{\text {tree }}$ and, therefore, $f(t=(g \phi / m)) \rightarrow 1+t$ in this limit of vanishing coupling. Now, because $t$ is arbitrary in this limit, $f(t)$ is thus evaluated to be given by $f(t)=1+t$ for all $t$. Therefore, the exact effective superpotential is found to be

$$
W_{\mathrm{eff}}=m \phi^{2}+g \phi^{3}=W_{\mathrm{tree}}
$$

i.e. the superpotential is not renormalized [10].

This argument rederives the standard perturbative non-renormalization theorem [16] and extends it beyond perturbation theory. Strictly speaking, the Wess-Zumino model probably does not exist as an interacting quantum field theory in four dimensions except as an effective low energy theory of a more fundamental theory and, therefore, this non-perturbative result is only of limited interest. This nonperturbative proof of the Wess-Zumino non-renormalization can be directly applied to two dimensions, where such models do exist as interacting quantum field theories.

If there are several fields, some light and some heavy, the heavy fields can be integrated out to yield a low energy effective Lagrangian for the light fields. The contribution of tree diagrams with intermediate heavy fields should be then included

in the effective action. The above simple rules allow such diagrams to contribute and are thus compatible with the known tree level renormalization of the superpotential.

\subsection{The 1PI effective action}

There are two different objects which are usually called "the effective action:" the 1PI effective action and the Wilsonian one. When there are no interacting massless particles, these two effective actions are identical. This is often the case 
in the Higgs or confining phases. However, when interacting massless particles are present, the 1PI effective action suffers from IR ambiguities and might suffer from holomorphic anomalies [17]. These are absent in the Wilsonian effective action.

Consider the theory with a tree level superpotential with sources for the gauge invariant polynomials $X^{r}$ in the matter fields, $W_{\text {tree }}=\sum_{r} g_{r} X^{r}$, with the $g_{r}$ regarded as background chiral superfield sources [10]. The functional integral with the added source terms gives the standard generating function for the correlation functions, $\Gamma(g)$. If supersymmetry is not broken, $\Gamma(g)$ is supersymmetric (otherwise we should include the Goldstino field and supersymmetry will be realized non-linearly) and $\Gamma(g)=\ldots+\int d^{2} \theta W_{L}(g)$. Using $W_{L}(g)$ we can compute the expectation values

$$
\frac{\partial W_{L}(g)}{\partial g_{r}}=\left\langle X^{r}\right\rangle \text {. }
$$

It is standard to perform a Legendre transform to find the 1PI effective action for the operators $X_{r}$ :

$$
W_{\mathrm{dyn}}\left(X^{r}\right)=\left(W_{L}\left(g_{r}\right)-\sum_{r} g_{r} X^{r}\right)_{\left\langle g_{r}\right\rangle},
$$

where the $\left\langle g_{r}\right\rangle$ are the solutions of (2.8). The transformation from $W_{L}\left(g_{r}\right)$ to $W_{\mathrm{dyn}}\left(X_{r}\right)$ can be inverted by the inverse Legendre transform as

$$
W_{L}(g)=\left(W_{\mathrm{dyn}}\left(X^{r}\right)+\sum_{r} g_{r} X^{r}\right)_{\left\langle X^{r}\right\rangle},
$$

where the $X^{r}$ are evaluated at their expectation values $\left\langle X^{r}\right\rangle$, which solve

$$
\frac{\partial W_{\mathrm{dyn}}}{\partial X_{r}}+g_{r}=0
$$

The 1PI effective superpotential

$$
W_{\text {eff }}(X, g)=W_{\mathrm{dyn}}\left(X^{r}\right)+\sum_{r} g_{r} X^{r}
$$

has the property that the equations of motion (2.11) for the fields $X^{r}$ derived from it determine their expectation values. In some cases the superpotential $W_{\text {eff }}$ obtained

1 In writing this expression we should think of the coupling constants $g_{r}$ as background superfields. Otherwise, $W_{L}(g)$ is a constant superpotential, which has no effect in global supersymmetry. Indeed, the following equation can be interpreted as differentiating the action with respect to the $F$ component of $g_{r}$. 
by the above Legendre transform is the same as the Wilsonian superpotential for the light fields. In applying this procedure we should be careful of the following pitfalls:

1. The theory with the sources should have a gap. Otherwise, the 1PI action is ill defined.

2. The theory with the sources might break supersymmetry. In that case $W_{L}$ is ill defined.

3. As the sources are turned off, some particles become massless. Their interpolating fields should be among the composite fields $X^{r}$. If some massless particles cannot be represented by a gauge invariant operator $X^{r}$, the effective superpotential derived this way will not include them. This often leads to singularities.

4. The theory might also have other branches which are present only when some sources vanish. In this case there are new massless particles at that point and the 1PI action might miss some of the branches. In other words, then the Legendre transform does not exist.

5. If some composites do not represent massless particles, they should be integrated out. Although we can use the effective superpotential to find their expectation values, we cannot think of them as fields corresponding to massive particles except near a point where they become massless.

There are known examplest of each of these situations; in these cases the 1PI effective action is misleading, failing to capture important aspects of the physics.

When we can use this procedure to find the Wilsonian action, the linearity of $W_{\text {eff }}$ (2.12) in the sources provides a derivation of the linearity of the Wilsonian effective action in the sources. See [19,20] for a related discussion.

This approach is particularly useful when we know how to compute $W_{L}\left(g_{r}\right)$

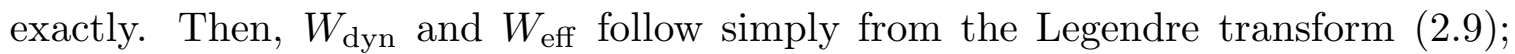
this is the "integrating in" discussed in [21,22]. One situation where $W_{L}\left(g_{r}\right)$ can be determined is when the $X^{r}$ are all quadratic in the elementary fields. In that

2 As a simple example of a situation in which the Legendre transform analysis is incomplete because supersymmetry is dynamically broken by the added source terms, consider $N=1$ supersymmetric $S U(2)$ with a single field $Q$ in the 4 of $S U(2)$ [18]. The theory without added source terms has a one complex dimensional smooth moduli space of vacua labeled by $\langle X\rangle$, where $X=Q^{4}$ is the basic gauge invariant, with a superpotential $W(X)=0$. Adding a source $W=g X$ does not lead to a supersymmetric effective superpotential $W(g)$ - rather, it breaks supersymmetry [18]. (As discussed in [18], it is also possible that there is a non-Abelian Coulomb phase at the origin of the moduli space and that supersymmetry is unbroken with the added source term. In that case the 1PI analysis again fails to capture the physics.) 
case, the sources $g_{r}$ are simply mass terms for the matter fields and $W_{L}(g)$ is the superpotential for the low energy gauge theory with the massive matter integrated out, expressed in terms of the quantities in the high-energy theory.

These issues will be exhibited and further discussed in later sections.

\subsection{Extended supersymmetry}

Theories with extended supersymmetry are further constrained. For example, $N=2$ supersymmetry combines an $N=1$ vector superfield with a chiral superfield $\Phi$ in the adjoint representation of the gauge group into an $N=2$ vector multiplet. In particular, $N=2$ supersymmetry includes a global $S U(2)_{R}$ symmetry which rotates the gluino component of an $N=1$ vector superfield into the fermion component of the chiral superfield. This symmetry relates the Kahler potential for the chiral superfield to the $\tau_{\text {eff }}$ in the kinetic term (2.2) for the gauge field. Therefore, the Kahler potential for chiral superfields which are part of $N=2$ vector multiplets are determined in terms of holomorphic functions and can thus often be obtained exactly.

Another type of $N=2$ supermultiplet is the hypermultiplet, consisting of two $N=1$ chiral multiplets $Q$ and $\widetilde{Q}$. The global $S U(2)_{R}$ symmetry rotates the scalar component of $Q$ into the scalar component of $\widetilde{Q}^{\dagger}$. This symmetry implies that the Kahler potential for these fields must yield a metric which is hyper-Kahler. $N=2$ supersymmetry further implies that this metric is not corrected by quantum effects.

Another condition on theories with $N=2$ supersymmetry is the Bogomol'nyiPrasad, Sommerfeld bound [23,24], which is related to the central term in the $N=2$ algebra [25]. The mass of any state satisfies

$$
M \geq \sqrt{2}|Z|,
$$

where $Z$ is the central term of the $N=2$ algebra, involving the gauge and global quantum numbers of the state. The BPS saturated states, for which the inequality (2.13) is saturated, are some of the stable states in the spectrum. Because $Z$ is a holomorphic object, it can often be exactly determined; (2.13) then yields the exact mass spectrum for some of the stable states.

This review will focus on $N=1$ supersymmetry. We refer the reader to the literature for further details about extended supersymmetry.

\section{Classical super gauge theories - classical moduli spaces of vacua}

The classical Lagrangian of a supersymmetric theory with gauge group $G$, matter superfields $\Phi_{f}$ in representations $R(f)$ of $G$, and zero tree level superpotential is

$$
\mathcal{L}=\mathcal{L}_{o}+\sum_{f, a} \phi_{f}^{\dagger} \lambda^{a} T_{f}^{a} \psi_{f}+\text { h.c. }+\sum_{a}\left(\sum_{f} \phi_{f}^{\dagger} T_{f}^{a} \phi_{f}\right)^{2},
$$


where $\mathcal{L}_{o}$ are the obvious gauge invariant kinetic terms for the gauge and matter fields and $T_{f}^{a}$ are the $G$ generators in representation $R(f)$. The interactions in (3.1) are related by supersymmetry to the coupling in $\mathcal{L}_{o}$ of gauge fields to matter.

Classical gauge theories often have "D-flat" directions of non-zero $\left\langle\phi_{f}\right\rangle$ along which the squark potential in (3.1) vanishes. In other words, these theories often have classical moduli spaces of degenerate vacua. As a simple example, consider $U(1)$ gauge theory with a matter superfield $Q$ of charge 1 and $\widetilde{Q}$ of charge -1 . The squark potential in (3.1) is $V=\left(Q^{\dagger} Q-\widetilde{Q}^{\dagger} \widetilde{Q}\right)^{2}$ and thus there is a continuum of degenerate vacua labeled, up to gauge equivalence, by $\langle Q\rangle=\langle\widetilde{Q}\rangle=a$, for any complex $a$. In vacua with $a \neq 0$ the gauge group is broken by the super Higgs mechanism. The gauge superfield gets mass $|a|$ by "eating" one chiral superfield degree of freedom from the matter fields. Since we started with the two superfields $Q$ and $\widetilde{Q}$, one superfield degree of freedom remains massless. The massless superfield can be given a gauge invariant description as $X=Q \widetilde{Q}$. In the vacuum labeled as above by $a,\langle X\rangle=a^{2}$. Because $a$ is arbitrary, there is no potential for $X$, $W_{c l}(X)=0$ - classically $X$ is a "modulus" field whose expectation value labels a classical moduli space of degenerate vacua. The classical Kahler potential of the microscopic theory is $K_{c l}=Q^{\dagger} e^{V} Q+\widetilde{Q}^{\dagger} e^{-V} \widetilde{Q}$. In terms of the light field $X$, $K_{c l}=2 \sqrt{X^{\dagger} X}$, which has a conical $\left(Z_{2}\right.$ orbifold) singularity at $X=0$. A singularity in a low energy effective action generally reflects the presence of additional massless fields which should be included in the effective action. Indeed, the singularity at $X=0$ corresponds to the fact that the gauge group is unbroken and all of the original microscopic fields are classically massless there.

As in the above simple example, the classical moduli space of vacua is the space of squark expectation values $\left\langle\phi_{f}\right\rangle$, modulo gauge equivalence, along which the potential in (3.1) vanishes. It can always be given a gauge invariant description in terms of the space of expectation values $\left\langle X_{r}\right\rangle$ of gauge invariant polynomials in the fields subject to any classical relations. This is because setting the potential in (3.1) to zero and modding out by the gauge group is equivalent to modding out by the complexified gauge group. The space of chiral superfields modulo the complexified gauge group can be parameterized by the gauge invariant polynomials modulo any classical relations. These results follow from results in geometrical invariant theory [26]; see also [27] for a recent discussion. As in the above example, the fields $X_{r}$ correspond to the matter fields left massless after the Higgs mechanism and are classical moduli, $W_{c l}\left(X_{r}\right)=0$.

The vacuum degeneracy of classical moduli spaces of vacua is not protected by any symmetry. In fact, vacua with different expectation values of the fields are physically inequivalent: as in the above example, the masses of the massive vector bosons depend on the expectation values $\left\langle X_{r}\right\rangle$. Therefore, the degeneracy of a classical moduli space of vacua is accidental and can be lifted in the quantum 
theory by a dynamically generated $W_{\text {eff }}\left(X_{r}\right)$. We will often be able to determine $W_{\text {eff }}\left(X_{r}\right)$ by the considerations discussed above.

\section{Example: SUSY QCD}

Consider supersymmetric $S U\left(N_{c}\right)$ gauge theory with $N_{f}$ quark flavors $Q^{i}$ in the fundamental representation and $\widetilde{Q}_{\tilde{i}}$ in the anti-fundamental, $i, \tilde{i}=1 \ldots N_{f}$. In the absence of mass terms, there is a classical moduli space of vacua given, up to gauge and global symmetry transformations, by:

$$
Q=\widetilde{Q}=\left(\begin{array}{llll}
a_{1} & & & \\
& a_{2} & & \\
& & \cdot & \\
& & & a_{N_{f}}
\end{array}\right)
$$

for $N_{f}<N_{c}$, with $a_{i}$ arbitrary, and by

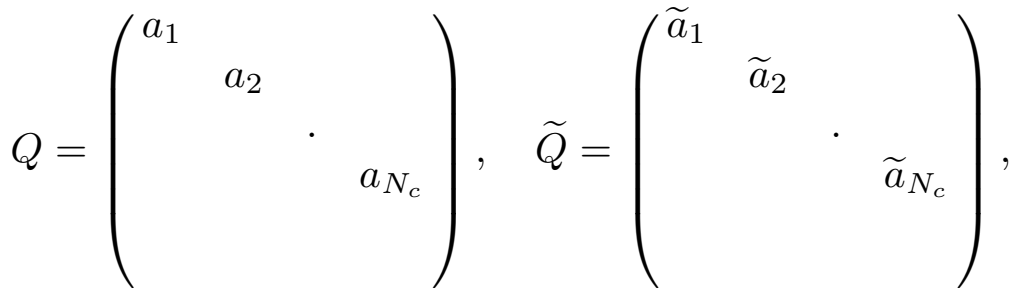

$$
\begin{aligned}
& \left|a_{i}\right|^{2}-\left|\widetilde{a}_{i}\right|^{2}=\text { independent of } i,
\end{aligned}
$$

for $N_{f} \geq N_{c}$.

For $N_{f}<N_{c}$, the gauge invariant description of the classical moduli space is in terms of arbitrary expectation values of the "mesons" $M_{\tilde{j}}^{i}=Q^{i} \widetilde{Q}_{\tilde{j}}$. For $N_{f} \geq N_{c}$, it is also possible to form "baryons" $B^{i_{1} \ldots i_{N_{c}}}=Q^{i_{1}} \ldots Q^{i_{N_{c}}}$ and $\widetilde{B}_{\tilde{i}_{1} \ldots \tilde{i}_{N_{c}}}=\widetilde{Q}_{\tilde{i}_{1}} \ldots \widetilde{Q}_{\tilde{i}_{N_{c}}}$. The gauge invariant description of the classical moduli space for $N_{f} \geq N_{c}$ is given in terms of the expectation values of $M, B$ and $\widetilde{B}$, subject to the following classical constraints. Up to global symmetry transformations, the expectation values are

$$
\begin{aligned}
& M=\left(\begin{array}{cccc}
a_{1} \widetilde{a}_{1} & & & \\
& a_{2} \widetilde{a}_{2} & & \\
& & a_{N_{c}} \widetilde{a}_{N_{c}} \\
& & &
\end{array}\right) \\
& B^{1, \ldots, N_{c}}=a_{1} a_{2} \ldots a_{N_{c}} \\
& \widetilde{B}_{1, \ldots, N_{c}}=\widetilde{a}_{1} \widetilde{a}_{2} \ldots \widetilde{a}_{N_{c}}
\end{aligned}
$$


with all other components of $M, B$ and $\widetilde{B}$ vanishing. Therefore, the rank of $M$ is at most $N_{c}$. If it is less than $N_{c}$, either $B=0$ with $\widetilde{B}$ having rank at most one or $\widetilde{B}=0$ with $B$ having rank at most one. If the rank of $M$ is equal to $N_{c}$, both $B$ and $\widetilde{B}$ have rank one and the product of their eigenvalues is the same as the product of non-zero eigenvalues of $M$.

As discussed above, the physical interpretation of the flat directions is that the gauge group is Higgsed. If $B=\widetilde{B}=0$ and $M$ has rank $k, S U\left(N_{c}\right)$ is broken to $S U\left(N_{c}-k\right)$ with $N_{f}-k$ light flavors.

Already at the classical level, we can integrate out the massive fields and consider an effective Lagrangian for the massless modes. Their expectation values label the particular ground state we expand around, and hence they are coordinates on the classical moduli space. The classical moduli space is not smooth. Its singularities are at the points of enhanced gauge symmetry. For instance, when $a_{i}=\widetilde{a}_{i}=0$ for every $i$ the gauge symmetry is totally unbroken. Therefore, the low energy effective theory of the moduli is singular there. This should not surprise us. At these singular points there are new massless particles - gluons. An effective Lagrangian without them is singular. If we include them in the low energy description, the Lagrangian is smooth.

In the next two sections we will see how this picture changes in the quantum theory. At large expectation values of the fields, far from the classical singularities, the gauge symmetry is broken at a high scale, the quantum theory is weakly coupled, and semi-classical techniques are reliable. We expect the quantum corrections to the classical picture to be small there. On the other hand, at small field strength the quantum theory is strongly coupled and the quantum corrections can be large and dramatically modify the classical behavior. In particular, the nature of the classical singularities, which are at strong coupling, is generally totally different in the quantum theory.

\section{SUSY QCD for $N_{f} \leq N_{c}+1$}

Because these theories have matter fields in the fundamental representation of the gauge group, as mentioned in the introduction, there is no invariant distinction

between the Higgs and the confining phases [2]. It is possible to smoothly interpolate from one interpretation to the other.

\section{1. $N_{f}<N_{c}-$ No Vacuum}

The first question to ask is whether the classical vacuum degeneracy can be lifted quantum mechanically by a dynamically generated superpotential. The form 
of such a superpotential is constrained by the symmetries. At the classical level, the symmetries are

$$
S U\left(N_{f}\right)_{L} \times S U\left(N_{f}\right)_{R} \times U(1)_{A} \times U(1)_{B} \times U(1)_{R}
$$

where the the quarks transform as

$$
\begin{aligned}
& Q \quad\left(N_{f}, 1,1,1, \frac{N_{f}-N_{c}}{N_{f}}\right) \\
& \widetilde{Q} \quad\left(1, \bar{N}_{f}, 1,-1, \frac{N_{f}-N_{c}}{N_{f}}\right) .
\end{aligned}
$$

$U(1)_{R}$ is an $R$ symmetry (the gauginos have charge +1 , the squark components of $Q$ and $\widetilde{Q}$ have the charge $R(Q)$ indicated above, and the charge of the fermion components is $R(\psi)=R(Q)-1)$. The charges were chosen so that only the symmetry $U(1)_{A}$ is anomalous in the quantum theory. Considering the anomalous $U(1)_{A}$ as explicitly broken by fermion zero modes in an instanton background, $U(1)_{A}$ leads to a selection rule. The instanton amplitude is proportional to $e^{-S_{\text {Inst. }}}=e^{-8 \pi^{2} g^{-2}(\mu)+i \theta}=(\Lambda / \mu)^{3 N_{c}-N_{f}}$, where $\Lambda$ is the dynamically generated scale of the theory, and we integrated the 1-loop beta function. Therefore, $U(1)_{A}$ is respected provided we assign $\Lambda^{3 N_{c}-N_{f}}$ charge $2 N_{f}$ to account for the charge of the fermion zero modes under $U(1)_{A}$. The dependence on the scale $\Lambda$ is thus determined by the $U(1)_{A}$ selection rule.

There is a unique superpotential which is compatible with these symmetries [28]

$$
W_{\text {eff }}=C_{N_{c}, N_{f}}\left(\frac{\Lambda^{3 N_{c}-N_{f}}}{\operatorname{det} \widetilde{Q} Q}\right)^{1 /\left(N_{c}-N_{f}\right)},
$$

where $C_{N_{c}, N_{f}}$ are constants which depend on the subtraction scheme. Therefore, if the vacuum degeneracy is lifted, this particular superpotential must be generated. For $N_{f} \geq N_{c}$ this superpotential does not exist (either the exponent diverges or the determinant vanishes) and therefore the vacuum degeneracy cannot be lifted. We will return to $N_{f} \geq N_{c}$ in the next subsections.

Note that the superpotential (4.3) is non-perturbative and is thus not in conflict with the perturbative non-renormalization theorem. Indeed, the above argument demonstrates that the perturbative non-renormalization and its non-perturbative violation can be understood simply as the need for obtaining a well-defined charge violation of the anomalous $U(1)_{A}$ as occurs, for example, in an instanton background.

The superpotential (4.3) is further constrained by considering various limits. For example, consider the limit of large $M_{\tilde{N}_{f}}^{N_{f}}$, i.e. large $a_{N_{f}}$ in (3.2), which breaks 
$S U\left(N_{c}\right)$ with $N_{f}$ flavors to $S U\left(N_{c}-1\right)$ with $N_{f}-1$ light flavors by the Higgs mechanism at energy $a_{N_{f}}$. Matching the running gauge coupling at energy $a_{N_{f}}$, the low energy theory has scale $\Lambda_{L}^{3\left(N_{c}-1\right)-\left(N_{f}-1\right)}=\Lambda^{3 N_{c}-N_{f}} / a_{N_{f}}^{2}$. The fact that the scales are so matched without any threshold factors reflects a choice of subtraction scheme; this is the correct matching, for example, in the $\overline{D R}$ scheme. Requiring (4.3) to properly reproduce the superpotential of the low energy theory in this limit gives $C_{N_{c}, N_{f}}=C_{N_{c}-N_{f}}$.

Next consider giving $Q_{N_{f}}$ and $\widetilde{Q}_{\tilde{N}_{f}}$ a large mass by adding $W_{\text {tree }}=m M_{\tilde{N}_{f}}^{N_{f}}$. The low energy theory is $S U\left(N_{c}\right)$ with $N_{f}-1$ flavors. Matching the running gauge coupling at the transition scale $m$, the low energy theory has scale $\Lambda_{L}^{3 N_{c}-\left(N_{f}-1\right)}=$ $m \Lambda^{3 N_{c}-N_{f}}$. Again, this equality is up to a scheme dependent threshold factor which is one in the $\overline{D R}$ scheme. Using the symmetries, the exact superpotential with the added mass term is of the form

$$
W_{\text {exact }}=\left(\frac{\Lambda^{3 N_{c}-N_{f}}}{\operatorname{det} M}\right)^{1 /\left(N_{c}-N_{f}\right)} f\left(t=m M_{\tilde{N}_{f}}^{N_{f}}\left(\frac{\Lambda^{3 N_{c}-N_{f}}}{\operatorname{det} M}\right)^{-1 /\left(N_{c}-N_{f}\right)}\right) .
$$

In the limit of small mass and weak coupling, we know that $f(t)=C_{N_{c}, N_{f}}+t$. Because all values of $t$ can be obtained in this limit, the function $f(t)$ is evaluated in this understood limit to be $f(t)=C_{N_{c}, N_{f}}+t$ for all $t$. The exact superpotential with the added mass term is thus

$$
W_{\text {exact }}=C_{N_{c}, N_{f}}\left(\frac{\Lambda^{3 N_{c}-N_{f}}}{\operatorname{det} M}\right)^{1 /\left(N_{c}-N_{f}\right)}+m M_{\tilde{N}_{f}}^{N_{f}} .
$$

Requiring (4.5) to give the correct superpotential in the low energy theory upon integrating out $M_{\tilde{N}_{f}}^{N_{f}}$ relates $C_{N_{c}, N_{f}}$ to $C_{N_{c}, N_{f}-1}$ which, when combined with $C_{N_{c}, N_{f}}=C_{N_{c}-N_{f}}$, determine that $C_{N_{c}, N_{f}}=\left(N_{c}-N_{f}\right) C^{1 /\left(N_{c}-N_{f}\right)}$ with $C$ a universal constant.

For $N_{f}=N_{c}-1$, the superpotential (4.3) is proportional to the one-instanton action and, thus, the constant $C$ can be exactly computed via a one-instanton calculation. Because the gauge group is completely broken by the Higgs mechanism for $N_{f}=N_{c}-1$ (for $\operatorname{det} M \neq 0$ ), the instanton calculation is reliable (there is no infra-red divergence). The universal constant $C$ can be computed by considering the particular case $N_{c}=2, N_{f}=1$. The direct instanton calculation [29] reveals that the constant $C \neq 0$. The more detailed analysis of [30] shows that $C=1 \mathrm{in}$ the $\overline{D R}$ scheme. For $N_{f}<N_{c}$ there is thus a dynamically generated superpotential

$$
W_{\mathrm{eff}}=\left(N_{c}-N_{f}\right)\left(\frac{\Lambda^{3 N_{c}-N_{f}}}{\operatorname{det} \widetilde{Q} Q}\right)^{1 /\left(N_{c}-N_{f}\right)} .
$$


While $W_{\text {eff }}$ is generated by instantons for $N_{f}=N_{c}-1$, for $N_{f}<N_{c}-1$ it is associated with gaugino condensation in the unbroken $S U\left(N_{c}-N_{f}\right)$ gauge group [29]. In particular, the low energy theory has a WZ term 3

$$
\int d^{2} \theta \log (\operatorname{det} M)\left(W_{\alpha} W^{\alpha}\right)_{S U\left(N_{c}-N_{f}\right)}=\left(M^{-1}\right)_{i}^{\tilde{j}} F_{M_{\tilde{j}}^{i}} \lambda_{\alpha} \lambda^{\alpha}+\ldots
$$

to account for the matching to the high energy theory. If gaugino condensation occurs in the low energy theory, $\langle\lambda \lambda\rangle=c \Lambda_{N_{c}-N_{f}}^{3}$ where $c$ is a constant and $\Lambda_{N_{c}-N_{f}}$ is the scale of the low energy $S U\left(N_{c}-N_{f}\right)$ gauge theory, given in terms of the scale $\Lambda$ of the high-energy theory by $\Lambda_{N_{c}-N_{f}}^{3\left(N_{c}-N_{f}\right)}=\Lambda^{3 N_{c}-N_{f}} / \operatorname{det} M$, (4.7) yields a term $c \Lambda_{N_{c}-N_{f}}^{3} M^{-1} F_{M}$ in the effective Lagrangian. Our superpotential (4.6) indeed gives exactly such a term, $\left(\Lambda^{3 N_{c}-N_{f}} / \operatorname{det} M\right)^{1 /\left(N_{c}-N_{f}\right)} M^{-1} F_{M}=\Lambda_{N_{c}-N_{f}}^{3} M^{-1} F_{M}$, in the effective Lagrangian. Therefore, gaugino condensation occurs in $N=1$ $S U\left(N_{c}-N_{f}\right)$ Yang-Mills theory with the normalization

$$
\left\langle\lambda_{\alpha} \lambda^{\alpha}\right\rangle=e^{2 \pi i k /\left(N_{c}-N_{f}\right)} \Lambda_{N_{c}-N_{f}}^{3} \quad \text { with } \quad k=1 \ldots\left(N_{c}-N_{f}\right),
$$

where we explicitly exhibit the phase. Using (4.6) (whose normalization follows from a well understood instanton calculation), we have derived gaugino condensation (4.8), including its normalization in the $\overline{D R}$ scheme, in the low energy $N=1$ Yang-Mills theory. (For related work on this model see also references [11] and [31].)

The dynamically generated superpotential (4.6) leads to a squark potential which slopes to zero for $\operatorname{det} M \rightarrow \infty$. Therefore, the quantum theory does not have a ground state. We started with an infinite set of vacua in the classical theory and ended up in the quantum theory without a vacuum!

Consider adding $W_{\text {tree }}=\operatorname{Tr} m M$, giving masses to the $N_{f}$ flavors. As in (4.5), symmetries and the weak coupling and small mass limit determine that the exact superpotential is $W_{\text {full }}=W_{\text {eff }}+W_{\text {tree }}$. The vacua are given by $\langle M\rangle$ solving $\left.\frac{\partial W_{\text {full }}}{\partial M_{\dot{j}}^{i}}\right|_{\langle M\rangle}=0$. This gives $N_{c}$ vacua

$$
\left\langle M_{\tilde{j}}^{i}\right\rangle=\left(\operatorname{det} m \Lambda^{3 N_{c}-N_{f}}\right)^{1 / N_{c}}\left(\frac{1}{m}\right)_{\tilde{j}}^{i},
$$

corresponding to the $N_{c}$ branches of the $N_{c}$-th root. For large $m$, the matter fields are very massive and decouple, leaving a low energy $S U\left(N_{c}\right)$ pure Yang-Mills theory. Indeed, the low energy theory has confinement with a mass gap and $N_{c}$ vacua.

3 We absorb a factor of $1 / 32 \pi^{2}$ into the definition of $W_{\alpha}^{2}$ 
Evaluating (4.6) with the expectation values (4.9) yields

$$
W_{L}(m)=N_{c}\left(\Lambda^{3 N_{c}-N_{f}} \operatorname{det} m\right)^{1 / N_{c}}=N_{c} \Lambda_{L}^{3},
$$

where $\Lambda_{L}$ is the scale of the low energy $S U\left(N_{c}\right)$ Yang- Mills theory. Expressed in terms of $\Lambda_{L}$, the superpotential (4.10) is interpreted as the result of gaugino condensation in the low energy $S U\left(N_{c}\right)$ Yang-Mills theory. Expressed in terms of $m$ and $\Lambda$, the superpotential (4.10) is the effective superpotential which yields the expectation values (4.9) via (2.8), $\frac{\partial W_{L}(m)}{\partial m}=\langle M\rangle$. As in (2.9), $W_{L}(m)$ leads to an effective superpotential for the operators $M$ :

$$
W_{\mathrm{dyn}}=\left(N_{c}-N_{f}\right)\left(\frac{\Lambda^{3 N_{c}-N_{f}}}{\operatorname{det} M}\right)^{1 /\left(N_{c}-N_{f}\right)} .
$$

In this case $W_{\text {dyn }}$ agrees with the Wilsonian effective superpotential (4.6), as could have been expected because this theory satisfies all of the conditions spelled out in sect. 2.3. The fact that the Wilsonian effective action is here the same as the 1PI effective action provides a simple alternate derivation of the linearity derived in (4.5).

It is also possible to "integrate in" operators which do not correspond to massless particles. Then, the effective action can be used only to compute their expectation values, rather than for studying them as massive particles. An example is the "glueball" field $S=-W_{\alpha}^{2}$, whose source is $\log \Lambda^{3 N_{c}-N_{f}}$. Integrating in $S$ by the Legendre transform of (4.11) with respect to the source $\log \Lambda^{3 N_{c}-N_{f}}$ yields

$$
W(S, M)=S\left[\log \left(\frac{\Lambda^{3 N_{c}-N_{f}}}{S^{N_{c}-N_{f}} \operatorname{det} M}\right)+\left(N_{c}-N_{f}\right)\right],
$$

the superpotential obtained in [32]. Adding mass terms $W_{\text {tree }}=\operatorname{Tr} m M$ and integrating out $M$ yields

$$
W(S)=S\left[\log \left(\frac{\Lambda_{L}^{3 N_{c}}}{S^{N_{c}}}\right)+N_{c}\right],
$$

where $\Lambda_{L}$ is the scale of the low energy $S U\left(N_{c}\right)$ Yang-Mills theory, $\Lambda_{L}^{3 N_{c}}=$ $\operatorname{det} m \Lambda^{3 N_{c}-N_{f}}$. This superpotential simply gives the information discussed above: supersymmetric $S U\left(N_{c}\right)$ Yang-Mills theory has $N_{c}$ supersymmetric vacua with the gaugino condensates (4.8) and superpotential $W=N_{c} \Lambda_{L}^{3}$. Working with such effective superpotentials which include massive fields can be convenient when interesting but complicated dynamics is encoded in the integrating out of these massive fields. Several such examples can be found in [21]. However, as stressed above, we should not think of $S$ as a field describing a massive particle. 
4.2. $N_{f}=N_{c}-$ Quantum moduli space with confinement with chiral symmetry breaking

As we said above, for $N_{f} \geq N_{c}$ the vacuum degeneracy can not be lifted. Therefore, the quantum theory also has a continuous space of inequivalent vacua. Since this space can be different than the classical one, we will refer to it as the "quantum moduli space." The most interesting questions about it are associated with the nature of its singularities. Classically, the singularities were associated with massless gluons. Are there singularities in the quantum moduli space? What massless particles are associated with those singularities?

The classical moduli space for $N_{f}=N_{c}$ is given in terms of the gauge invariant description (3.4) as the the space of expectation values of mesons $M_{\tilde{j}}^{i}$, and baryons $B$ and $\widetilde{B}$ subject to the classical constraint

$$
\operatorname{det} M-\widetilde{B} B=0,
$$

which follows from Bose statistics of $Q$ and $\widetilde{Q}$. This space has a singular submanifold $B=\widetilde{B}=0$ and $\operatorname{rank}(M) \leq N_{c}-2$, where $d(\operatorname{det} M-B \widetilde{B})=0$. Physically, the classical singularities reflect the fact that there are additional degrees of freedom, $S U\left(N_{c}-\operatorname{rank}(M)\right)$ gluons, on this submanifold.

The quantum moduli space is parameterized by the same fields but the constraint is modified [33] to

$$
\operatorname{det} M-\widetilde{B} B=\Lambda^{2 N_{c}} .
$$

This can be seen by adding mass terms $W_{\text {tree }}=\operatorname{Tr} m M$ and taking $m \rightarrow 0$. It follows from (4.9) that $\operatorname{det}\langle M\rangle=\Lambda^{2 N_{c}}$, independent of $m$, for $N_{f}=N_{c}$. This agrees with (4.15) for $\langle B \widetilde{B}\rangle=0$, which is the case with only added mass terms. Considering more generally $W_{\text {tree }}=\operatorname{Tr} m M+b B+\tilde{b} \widetilde{B}$ with $m, b, \tilde{b} \rightarrow 0,\langle B \widetilde{B}\rangle$ can be non-zero and the expectation values are found to satisfy (4.15) [22]. Because the right hand side of (4.15) is proportional to the one-instanton action, the quantum modification of the classical constraint is exactly given by a one-instanton contribution.

There are no singularities on the quantum moduli space given by (4.15) - all of the classical singularities have been smoothed out by quantum effects. As a similar but simpler example of the deformed moduli space, consider the space $X Y=\mu$ in $C^{2}$. For $\mu=0$ the space is a pair of cones (corresponding to the $X$ plane and the $Y$ plane) touching at their tips; the space is singular at the origin, where the cones touch. In the deformed space $\mu \neq 0$, the two cones are smoothed out to a single sheeted hyperboloid. It asymptotes to the two cones far from the origin but has a smooth hourglass shape where they connect. Similarly, the quantum space (4.15) is smooth, which is dramatically different from the classical space (4.14) near the origin. For large expectation values $M, B$ and $\widetilde{B}$, the difference becomes negligible, as it should be in the weak coupling region. 
Since there are no singularities on the quantum moduli space, the only massless particles are the moduli, the fluctuations of $M, B$, and $\widetilde{B}$ preserving (4.15). In the semi-classical region of large fields it is appropriate to think of the theory as "Higgsed." Near the origin, because the quantum theory is smooth in terms of the mesons and baryons, it is appropriate to think of the theory as being confining. There is a smooth transition from the region where a Higgs description is more appropriate to the region where a confining description is more appropriate. Again, this is possible because of the presence of matter fields in the fundamental representation of the gauge group [2].

Because the origin $M=B=\widetilde{B}=0$ is not on the quantum moduli space (4.15), the quantum dynamics necessarily break the anomaly free, chiral $S U\left(N_{f}\right) \times$ $S U\left(N_{f}\right) \times U(1)_{B} \times U(1)_{R}$ symmetry in (4.1). Different points on the quantum moduli space exhibit different patterns of chiral symmetry breaking. For example at $M_{\tilde{j}}^{i}=\Lambda^{2} \delta_{\tilde{j}}^{i}, B=\widetilde{B}=0$ the symmetry is broken as

$$
S U\left(N_{f}\right)_{L} \times S U\left(N_{f}\right)_{R} \times U(1)_{B} \times U(1)_{R} \rightarrow S U\left(N_{f}\right)_{V} \times U(1)_{B} \times U(1)_{R} .
$$

At $M=0, B=-\widetilde{B}=\Lambda^{N_{c}}$ the breaking pattern is

$$
S U\left(N_{f}\right)_{L} \times S U\left(N_{f}\right)_{R} \times U(1)_{B} \times U(1)_{R} \rightarrow S U\left(N_{f}\right)_{L} \times S U\left(N_{f}\right)_{R} \times U(1)_{R} .
$$

Some of the moduli are Goldstone bosons of the broken symmetry whereas others take the theory to vacua with different breaking patterns. For no vacuum in the quantum moduli space (4.15) is the full chiral symmetry unbroken. It is straightforward to check that the massless fermion spectrum, consisting of the fermionic components of the chiral superfield moduli, satisfies the 'tHooft anomaly conditions for the unbroken symmetries.

The constraint (4.15) can be implemented with a superpotential $W=A(\operatorname{det} M-$ $\left.B \widetilde{B}-\Lambda^{2 N_{c}}\right)$, with $A$ a Lagrange multiplier. The reader can verify that, upon adding $W_{\text {tree }}=m M_{\tilde{N}_{c}}^{N_{c}}$ to give a mass to the $N_{c}$-th flavor, the low energy theory with $N_{f}=N_{c}-1$ light flavors has the appropriate superpotential (4.6).

\section{3. $N_{f}=N_{c}+1$ - Confinement without chiral symmetry breaking}

We now add another massless flavor to the previous case. The classical moduli space is again described as in (3.4) by the mesons $M$, baryons $B_{i}=$ $\epsilon_{i j_{1} \ldots j_{N_{c}}} Q^{j_{1}} \ldots Q^{j_{N_{c}}}$ and $\widetilde{B} \tilde{i}=\epsilon^{\tilde{i} \tilde{j}_{1} \ldots \tilde{j}_{N_{c}}} \widetilde{Q}_{\tilde{j}_{1}} \ldots \widetilde{Q}_{\tilde{j}_{N_{c}}}$ subject to the constraints

$$
\begin{aligned}
& \operatorname{det} M\left(\frac{1}{M}\right)_{i}^{\tilde{j}}-B_{i} \widetilde{B}^{\tilde{j}}=0 \\
& M_{\tilde{j}}^{i} B_{i}=M_{\tilde{j}}^{i} \widetilde{B}^{\tilde{j}}=0 .
\end{aligned}
$$


Unlike the previous case, for $N_{f}=N_{c}+1$ the quantum moduli space is the same as the classical one 33. This can be seen by adding $W_{\text {tree }}=\operatorname{Tr} m M$, giving masses to all $N_{f}+1$ flavors. The expectation values of $M$ are then given by (4.9). By taking the limit $m_{i}^{\tilde{j}} \rightarrow 0$ in different ratios, it is found that $\left\langle M_{\tilde{j}}^{i}\right\rangle$ can be anywhere on the classical moduli space (4.18) of vacua.

Because the quantum moduli space of vacua is the same as the classical moduli space, it has singularities at strong coupling. The singularities, however, are interpreted differently than in the classical theory. Rather than being associated with massless gluons, the singularities are associated with additional massless mesons and baryons! In particular, at the point $M=\widetilde{B}=B=0$ the global chiral symmetry

$$
S U\left(N_{f}\right)_{L} \times S U\left(N_{f}\right)_{R} \times U(1)_{B} \times U(1)_{R}
$$

is unbroken and all the components of $M, B$ and $\widetilde{B}$ are massless and physical [33]. It is a non-trivial consistency check that this massless spectrum at the origin satisfies the 'tHooft anomaly matching conditions for the full global symmetry (4.19).

Away from the origin, all the degrees of freedom in $M, B$ and $\widetilde{B}$ are physical and they couple through the superpotential [33]

$$
W_{\text {eff }}=\frac{1}{\Lambda^{2 N_{c}-1}}\left(M_{\tilde{j}}^{i} B_{i} \widetilde{B}^{\tilde{j}}-\operatorname{det} M\right) .
$$

The classical constraints (4.18) appear as the equations of motion $\frac{\partial W_{\text {eff }}}{\partial M}=\frac{\partial W_{\text {eff }}}{\partial B}=$ $\frac{\partial W_{\text {eff }}}{\partial \overparen{B}}=0$. Far from the origin, in the region of weak coupling, the number of independent massless fields is the same as in the classical theory because the components of $M, B$, and $\widetilde{B}$ which are classically constrained get a large mass from $(4.20)$.

We conclude that the spectrum at the origin of field space consists of massless composite mesons and baryons and that the chiral symmetry of the theory is unbroken there. This is confinement without chiral symmetry breaking. Again, we see a smooth transition [2] from the semi-classical region where a Higgs description is more appropriate to a strongly coupled region where a confining description is more appropriate.

The reader can verify that adding $W_{\text {tree }}=m M_{N_{c}+1}^{N_{c}+1}$ to 4.20 to give a mass to the $N_{c}+1$-th flavor yields the quantum moduli space with constraint (4.15) in the low energy theory with $N_{f}=N_{c}$ light flavors.

\section{SUSY QCD for $N_{f}>N_{c}+1$}

\section{1. $N_{f} \geq 3 N_{c}$}

In this range the theory is not asymptotically free. This means that, because of screening, the coupling constant becomes smaller at large distances. Therefore, 
the spectrum of the theory at large distance can be read off from the Lagrangian - it consists of the elementary quarks and gluons. The long distance behavior of the potential between external electric test charges has the free electric behavior in (1.1). For this range of $N_{f}$, the theory is in a non-Abelian free electric phase.

We should add here that, strictly speaking, such a theory is not well defined as an interacting quantum field theory because of the Landau pole at $R \sim \Lambda^{-1}$. However, it can be a consistent description of the low energy limit of another theory.

\section{2. $\frac{3}{2} N_{c}<N_{f}<3 N_{c}$; Interacting non-Abelian Coulomb phase}

In this range the theory is asymptotically free. This means that at short distance the coupling constant is small and it becomes larger at longer distances. However, for this range of $N_{f}$ [33,8], rather than growing to infinity, it reaches a finite value - a fixed point of the renormalization group.

The exact beta function in supersymmetric QCD satisfies [34,17]

$$
\begin{aligned}
\beta(g) & =-\frac{g^{3}}{16 \pi^{2}} \frac{3 N_{c}-N_{f}+N_{f} \gamma\left(g^{2}\right)}{1-N_{c} \frac{g^{2}}{8 \pi^{2}}} \\
\gamma\left(g^{2}\right) & =-\frac{g^{2}}{8 \pi^{2}} \frac{N_{c}^{2}-1}{N_{c}}+\mathcal{O}\left(g^{4}\right),
\end{aligned}
$$

where $\gamma\left(g^{2}\right)$ is the anomalous dimension of the mass. Since there are values of $N_{f}$ and $N_{c}$ where the one loop beta function is negative but the two loop contribution is positive, there might be a non-trivial fixed point [35. Indeed, by taking $N_{c}$ and $N_{f}$ to infinity holding $N_{c} g^{2}$ and $\frac{N_{f}}{N_{c}}=3-\epsilon$ fixed, one can establish the existence of a zero of the beta function at $N_{c} g_{*}^{2}=\frac{8 \pi^{2}}{3} \epsilon+\mathcal{O}\left(\epsilon^{2}\right)$. Therefore, at least for large $N_{c}$ and $\epsilon=3-\frac{N_{f}}{N_{c}} \ll 1$, there is a non-trivial fixed point. It was argued in [8] that such a fixed point exists for every $\frac{3}{2} N_{c}<N_{f}<3 N_{c}$.

Therefore, for this range of $N_{f}$, the infrared theory is a non-trivial four dimensional superconformal field theory. The elementary quarks and gluons are not confined but appear as interacting massless particles. The potential between external electric sources behaves as

$$
V \sim \frac{1}{R}
$$

and therefore we refer to this phase of the theory as the non-Abelian Coulomb phase.

Given that such a fixed point exists, we can use the superconformal algebra to derive some exact results about the theory. This algebra includes an $R$ symmetry. It follows from the algebra that the dimensions of the operators satisfy

$$
D \geq \frac{3}{2}|R|
$$


the inequality is saturated for chiral operators, for which $D=\frac{3}{2} R$, and for anti-chiral operators, for which $D=-\frac{3}{2} R$. Exactly as in $\mathrm{N}=2$ theories in two dimensions, this has important consequences. Consider the operator product of two chiral operators, $\mathcal{O}_{1}(x) \mathcal{O}_{2}(0)$. All the operators in the resulting expansion have $R=R\left(\mathcal{O}_{1}\right)+R\left(\mathcal{O}_{2}\right)$ and hence $D \geq D\left(\mathcal{O}_{1}\right)+D\left(\mathcal{O}_{2}\right)$. Therefore, there is no singularity in the expansion at $x=0$ and we can define the product of the two operators by simply taking the limit of $x$ to zero. If this limit does not vanish, it leads to a new chiral operator $\mathcal{O}_{3}$ whose dimension is $D\left(\mathcal{O}_{3}\right)=D\left(\mathcal{O}_{1}\right)+D\left(\mathcal{O}_{2}\right)$. We conclude that the chiral operators form a ring.

The $R$ symmetry of the superconformal fixed point is not anomalous and commutes with the flavor $S U\left(N_{f}\right) \times S U\left(N_{f}\right) \times U(1)_{B}$ symmetry. Therefore, it must be the anomaly free $R$ symmetry appearing in (4.2). Hence the gauge invariant operators $\widetilde{Q} Q$ have 8

$$
D(\widetilde{Q} Q)=\frac{3}{2} R(\widetilde{Q} Q)=3 \frac{N_{f}-N_{c}}{N_{f}}
$$

and similarly

$$
D(B)=D(\widetilde{B})=\frac{3 N_{c}\left(N_{f}-N_{c}\right)}{2 N_{f}} .
$$

The value of $D(\widetilde{Q} Q)$ also follows from (5.1) - at the zero of the beta function $\gamma=-3 \frac{N_{c}}{N_{f}}+1$ and hence $D=\gamma+2=3 \frac{N_{f}-N_{c}}{N_{f}}$.

All of the gauge invariant operators at the infrared fixed point should be in unitary representations of the superconformal algebra. The complete list of such representations was given in [36] by extending the analysis [37] of the ordinary conformal algebra. One of the constraints on the representations which follows already from the analysis of [37] is that spinless operators have $D \geq 1$ (except the identity operator with $D=0$ ) and the bound is saturated for free fields (satisfying $\left.\partial_{\mu} \partial^{\mu} \Phi=0\right)$. For $D<1(D \neq 0)$ a highest weight representation includes a negative norm state which cannot exist in a unitary theory.

The fixed point coupling $g_{*}$ gets larger as the number of flavors is reduced. For $N_{f}$ at or below $\frac{3}{2} N_{c}$ the theory is very strongly coupled and goes over to a new phase, different from the interacting non-Abelian Coulomb phase. To see that the theory must be in a different phase, note that the value of $D(\widetilde{Q} Q)$ in (5.3) is inconsistent with the unitarity bound $D \geq 1$ for $N_{f}<\frac{3}{2} N_{c}$. The new phase will be explained below. A clue is the fact that the dimension of $M=\widetilde{Q} Q$ becomes one for $N_{f}=\frac{3}{2} N_{c}$, which shows that $M$ becomes a free field, i.e. $\partial^{2} M=0$. This suggests that in the correct description for $N_{f}=\frac{3}{2} N_{c}$ the field $M$, and perhaps even the whole IR theory, is free. 


\subsection{Duality}

The physics of the interacting fixed point obtained for the range $\frac{3}{2} N_{c}<N_{f}<$ $3 N_{c}$ has an equivalent, "magnetic," description [8]. It is based on the gauge group $S U\left(N_{f}-N_{c}\right)$, with $N_{f}$ flavors of quarks $q_{i}$ and $\widetilde{q}^{\tilde{i}}$ and gauge invariant fields $M_{\tilde{j}}^{i}$ with a superpotential

$$
W=\frac{1}{\mu} M_{\tilde{j}}^{i} q_{i} \tilde{q}^{\tilde{j}} .
$$

We will refer to this gauge group as the magnetic gauge group and to its quarks as magnetic quarks. Without the superpotential (5.5), the magnetic theory also flows to a non-Abelian Coulomb phase fixed point because $\frac{3}{2}\left(N_{f}-N_{c}\right)<N_{f}<3\left(N_{f}-N_{c}\right)$ for the above range of $N_{f}$. At this fixed point $M$ is a free field of dimension one and, using (5.3), $D(q \widetilde{q})=3 N_{c} / N_{f}$. Because the dimensions of chiral operators add, the superpotential (5.5) has dimension $D=1+3 N_{c} / N_{f}<3$ at the fixed point of the magnetic gauge theory and is thus a relevant perturbation, driving the theory to a new fixed point. The surprising fact is that this new fixed point is identical to that of the original, "electric," $S U\left(N_{c}\right)$ theory. Note that the two theories have different gauge groups and different numbers of interacting particles. Nevertheless, they describe the same fixed point. In other words, there is no experimental way to determine whether the $\frac{1}{R}$ potential between external sources is mediated by the interacting electric or the interacting magnetic variables. Such a phenomenon of two different Lagrangians describing the same long distance physics is common in two dimensions and is known there as quantum equivalence. These four dimensional examples generalize the duality [3] in finite $N=4$ supersymmetric theories [4] and in finite $N=2$ theories [5] to asymptotically free $N=1$ theories.

The scale $\mu$ in (5.5) is needed for the following reason. In the electric description $M_{\tilde{j}}^{i}=Q^{i} \widetilde{Q}_{\tilde{j}}$ has dimension two at the UV fixed point and acquires anomalous dimension (5.3) at the IR fixed point. In the magnetic description, $M_{m}$ is an elementary field of dimension one at the UV fixed point which flows to the same operator with dimension (5.3) at the IR fixed point. In order to relate $M_{m}$ to $M$ of the electric description in the UV, a scale $\mu$ must be introduced with the relation $M=\mu M_{m}$. Below we will write all the expressions in terms of $M$ and $\mu$ rather than in terms of $M_{m}$.

The magnetic theory has a scale $\widetilde{\Lambda}$ which is related to the scale $\Lambda$ of the electric theory by

$$
\Lambda^{3 N_{c}-N_{f}} \widetilde{\Lambda}^{3\left(N_{f}-N_{c}\right)-N_{f}}=(-1)^{N_{f}-N_{c}} \mu^{N_{f}},
$$

where $\mu$ is the dimensionful scale explained above. This relation of the scales has several consequences:

1. It is easy to check that it is preserved under mass deformations and along the flat directions (more details will be given below). The phase $(-1)^{N_{f}-N_{c}}$ is important 
in order to ensure that this is the case.

2. It shows that as the electric theory becomes stronger the magnetic theory becomes weaker and vice versa. It is the analog of $g \rightarrow 1 / g$ for asymptotically free theories.

3. Differentiating the action with respect to $\log \Lambda$ relates the field strengths of the electric and the magnetic theories as $W_{\alpha}^{2}=-\widetilde{W}_{\alpha}^{2}$. The minus sign in this expression is common in electric magnetic duality, which maps $E^{2}-B^{2}=-\left(\widetilde{E}^{2}-\right.$ $\left.\widetilde{B}^{2}\right)$. In our case it shows that the gluino bilinear in the electric and the magnetic theories are related by $\lambda \lambda=-\tilde{\lambda} \tilde{\lambda}$.

4. Because of the phase $(-1)^{N_{f}-N_{c}}$, the relation (5.6) does not look dual - if we perform another duality transformation it becomes $\Lambda^{3 N_{c}-N_{f}} \widetilde{\Lambda}^{3\left(N_{f}-N_{c}\right)-N_{f}}=$ $(-1)^{N_{c}} \tilde{\mu}^{N_{f}}$ and therefore

$$
\tilde{\mu}=-\mu \text {. }
$$

This minus sign is important when we dualize again. The dual of the dual magnetic theory is an $S U\left(N_{c}\right)$ theory with scale $\Lambda$, quarks $d^{i}$ and $\widetilde{d}_{\tilde{j}}$, and additional singlets $M_{\tilde{j}}^{i}$ and $N_{i}^{\tilde{j}}=q_{i} \widetilde{q}^{\tilde{j}}$, with superpotential

$$
W=\frac{1}{\tilde{\mu}} N_{i}^{\tilde{j}} d^{i} \widetilde{d}_{\tilde{j}}+\frac{1}{\mu} M_{\tilde{j}}^{i} N_{i}^{\tilde{j}}=\frac{1}{\mu} N_{i}^{\tilde{j}}\left(-d^{i} \widetilde{d}_{\tilde{j}}+M_{\tilde{j}}^{i}\right) .
$$

The first term is our standard superpotential of duality transformations $A$ and the second term is simply copied from (5.5). $M$ and $N$ are massive and can be integrated out using their equations of motion: $N=0, M_{\tilde{j}}^{i}=d^{i} \widetilde{d}_{\tilde{j}}$. This last relation shows that the quarks $d$ and $\widetilde{d}$ can be identified with the original electric quarks $Q$ and $\widetilde{Q}$. The dual of the magnetic theory is the original electric theory. The electric and magnetic theories have different gauge symmetries. This is possible because gauge symmetries really have to do with a redundant description of the physics rather than with symmetry. There is no problem with having two different redundant descriptions of the same physics. On the other hand, global symmetries are physical and should be the same in the electric and magnetic theories. Indeed, the magnetic theory has the same anomaly free global $S U\left(N_{f}\right) \times S U\left(N_{f}\right) \times U(1)_{B} \times U(1)_{R}$ as the electric theory, with the singlet $M_{\tilde{j}}^{i}$ transforming as $Q^{i} \widetilde{Q}_{\tilde{j}}$ and the magnetic quarks transforming as

$$
\begin{array}{ll}
q & \text { in } \quad\left(\bar{N}_{f}, 1, \frac{N_{c}}{N_{f}-N_{c}}, \frac{N_{c}}{N_{f}}\right) \\
\widetilde{q} & \text { in } \quad\left(1, N_{f},-\frac{N_{c}}{N_{f}-N_{c}}, \frac{N_{c}}{N_{f}}\right) .
\end{array}
$$

4 The relative minus sign between it and (5.5), which follows from (5.7), is common in Fourier or Legendre transforms. (Compare with (2.9) and (2.10).) 
This symmetry is anomaly free in the magnetic theory and it is preserved by the superpotential (5.5). Furthermore, the magnetic spectrum with these charges satisfies the 'tHooft anomaly matching conditions.

In order for the dual theory to describe the same physics as the electric theory, there must be a mapping of all gauge invariant primary operators of the electric theory to those of the dual theory. For example, the electric mesons $M_{\tilde{j}}^{i}=Q^{i} \widetilde{Q}_{\tilde{j}}$ and the singlets $M_{\tilde{j}}^{i}$ of the magnetic theory become identical in the infrared. All such mappings must be compatible with the global symmetry charges discussed above. Another set of gauge invariant operators of the electric theory are the baryons $B^{i_{1} \ldots i_{N_{c}}}=Q^{i_{1}} \cdots Q^{i_{N_{c}}}$ and $\widetilde{B}_{\tilde{i}_{1} \ldots \tilde{i}_{N_{c}}}=\widetilde{Q}_{\tilde{i}_{1}} \cdots \widetilde{Q}_{\tilde{i}_{N_{c}}}$. In the magnetic theory we can similarly form the baryons $b_{i_{1} \ldots i_{\tilde{N}_{c}}}=q_{i_{1}} \cdots q_{\tilde{N}_{c}}$ and $\widetilde{b}^{\tilde{i}_{1} \ldots \tilde{i}_{\tilde{N}_{c}}}=\tilde{q}^{\tilde{i}_{1}} \cdots \tilde{q}^{\tilde{i}_{\tilde{N}_{c}}}$, where $\tilde{N}_{c}=N_{f}-N_{c}$. At the fixed point, these operators are related via

$$
\begin{aligned}
B^{i_{1} \ldots i_{N_{c}}} & =C \epsilon^{i_{1} \ldots i_{N_{c}} j_{1} \ldots j_{N_{c}}} b_{j_{1} \ldots j_{\tilde{N}_{c}}}, \\
\widetilde{B}_{\tilde{i}_{1} \ldots \tilde{i}_{N_{c}}} & =C \epsilon_{\tilde{i}_{1} \ldots \tilde{i}_{N_{c}} \tilde{j}_{1} \ldots \tilde{j}_{\tilde{N}_{c}}} \widetilde{b}^{\tilde{j}_{1} \ldots \tilde{j}_{\tilde{N}_{c}}},
\end{aligned}
$$

with $C=\sqrt{-(-\mu)^{N_{c}-N_{f}} \Lambda^{3 N_{c}-N_{f}}}$. Note that these mappings respect the global symmetries discussed above. The normalization constant $C$ was fixed by symmetries and by limits to be discussed below. It follows from (5.6) that (5.10) respects the $Z_{2}$ nature of the duality.

$$
\text { 5.4. } N_{c}+2 \leq N_{f} \leq \frac{3}{2} N_{c}
$$

Recall that the electric $S U\left(N_{c}\right)$ theory with $N_{f}$ flavors was in a non-Abelian free electric phase for $N_{f} \geq 3 N_{c}$ and in the non-Abelian Coulomb phase for $\frac{3}{2} N_{c}<N_{f}<$ $3 N_{c}$, with the fixed point at larger electric coupling for smaller $N_{f}$. In the magnetic description of the non-Abelian Coulomb phase fixed point, the magnetic coupling at the fixed point is small for $N_{f}$ near $3\left(N_{f}-N_{c}\right)$ and gets larger with $3\left(N_{f}-N_{c}\right)-N_{f}$; in the magnetic description the fixed point is at weaker coupling for smaller $N_{f}$. It was seen that for $N_{f} \leq \frac{3}{2} N_{c}$ the theory must be in a different phase. In the magnetic description, the situation is clear: since $3\left(N_{f}-N_{c}\right) \leq N_{f}$, the magnetic $S U\left(N_{f}-N_{c}\right)$ gauge theory is not asymptotically free (and the added superpotential (5.5) is irrelevant) and thus weakly coupled at large distances. Therefore, the low energy spectrum of the theory consists of the $S U\left(N_{f}-N_{c}\right)$ gauge fields and the fields $M, q$, and $\widetilde{q}$ in the dual magnetic Lagrangian [8]. These magnetic massless states are composites of the elementary electric degrees of freedom. The massless composite gauge fields exhibit gauge invariance which is not visible in the underlying electric description. The theory generates new gauge invariance! Because there are massless magnetically charged fields, the theory is in a non-Abelian free magnetic phase. 


\subsection{Deformations of the dual theories}

We now consider deforming the theories, showing in detail how the electricmagnetic duality exchanges strong coupling with weak coupling and Higgs with confinement.

Consider starting from the electric theory and giving a mass to the $N_{f}$-th flavor by adding $W_{\text {tree }}=m M_{\tilde{N}_{f}}^{N_{f}}$. The low energy theory has $N_{f}-1$ light flavors and a scale $\Lambda_{L}$ related to the scale $\Lambda$ of the high energy theory by $\Lambda_{L}^{3 N_{c}-\left(N_{f}-1\right)}=m \Lambda^{3 N_{c}-N_{f}}$. As expected, the low energy electric theory is at stronger coupling; the mass term is a relevant operator, sending the theory to a more strongly coupled fixed point.

In the magnetic theory, adding $W_{\text {tree }}$ gives

$$
W=\frac{1}{\mu} M_{\tilde{i}}^{i} q_{i} \tilde{q}^{\tilde{i}}+m M_{\tilde{N}_{f}}^{N_{f}}
$$

The equations of motion of $M_{\tilde{N}_{f}}^{N_{f}}, M_{\tilde{N}_{f}}^{i}$ and $M_{\tilde{i}}^{N_{f}}$ lead to

$$
q_{N_{f}} \widetilde{q}^{\tilde{N}_{f}}=-\mu m, \quad q_{i} \widetilde{q}^{\tilde{N}_{f}}=q_{N_{f}} \widetilde{q}^{\tilde{i}}=0,
$$

(color indices are suppressed and summed) which show that the magnetic gauge group is broken by the Higgs mechanism to $S U\left(N_{f}-N_{c}-1\right)$ with $N_{f}-1$ quarks left massless. The equations of motion of the massive quarks lead to

$$
M_{\tilde{N}_{f}}^{N_{f}}=M_{\tilde{N}_{f}}^{i}=M_{\tilde{i}}^{N_{f}}=0
$$

The low energy superpotential is

$$
W=\frac{1}{\mu} \widehat{M}_{\tilde{i}}^{i} \hat{q}_{i} \hat{\widetilde{q}}^{\tilde{i}}, \quad i, \tilde{i}=1, \ldots, N_{f}-1
$$

where $\widehat{M}, \hat{q}$ and $\hat{\widetilde{q}}$ are the light fields with $N_{f}-N_{c}-1$ colors and $N_{f}-1$ flavors. The scale of the low energy magnetic theory is given by $\widetilde{\Lambda}_{L}^{3\left(\widetilde{N}_{c}-1\right)-\left(N_{f}-1\right)}=$ $\widetilde{\Lambda}^{3 \widetilde{N}_{c}-N_{f}} /\left\langle q_{N_{f}} \widetilde{q}^{\tilde{N}_{f}}\right\rangle$. Note that (5.6) and (5.10) are preserved in the low energy theories. The low energy magnetic theory is at weaker coupling and is the dual of the low energy electric theory. The duality is preserved under the mass term deformation and exchanges a more strongly coupled electric description of the new fixed point with a more weakly coupled magnetic description of the new fixed point.

The above discussion of the mass term deformation is incomplete for $N_{f}=N_{c}+2$, where the mass term for the $\left(N_{c}+2\right)$-th flavor triggers complete breaking of the magnetic gauge group. The low energy theory contains the mesons $\widehat{M}_{\tilde{i}}^{j}$, where the 
hat refers to the flavors $i, \tilde{i}=1 \ldots N_{c}+1$, and the singlets $\hat{q}_{i}$ and $\hat{\widetilde{q}}^{\tilde{i}}$, which are the components of the magnetic quarks remaining massless after the Higgs mechanism, with a superpotential as in (5.14). The map (5.10) for $N_{f}=N_{c}+1$ implies that the singlets $\hat{q}_{i}$ and $\hat{\widetilde{q}}^{\tilde{i}}$ are, in fact, the baryons $B_{i}$ and $\widetilde{B}^{i}$ of the low energy electric theory. It has already been suggested, at least for large $N_{c}$, that baryons can be thought of as solitons in the pion Lagrangian [38]. Here we see an explicit realization of a related idea - the baryons are magnetic monopoles of the elementary quarks and gluons! Taking the normalization in (5.10) into account, the superpotential (5.14) obtained in the low energy theory from flowing down from that of the magnetic, $N_{f}=N_{c}+2, S U(2)$ theory is

$$
W_{o}=\frac{1}{\Lambda_{L}^{2 N_{c}-1}} \widehat{M}_{\tilde{j}}^{i} B_{i} \widetilde{B}^{\tilde{j}}
$$

where $\Lambda_{L}$ is the scale of the low energy electric theory with $N_{f}=N_{c}+1$ light flavors. However, because the magnetic theory is completely Higgsed in the flow down from $N_{f}=N_{c}+2$, the low energy theory also includes instanton contributions in the broken magnetic group. In particular, the superpotential has an additional term which is the magnetic analog of (4.6)

$$
W_{\text {inst }}=\frac{\widetilde{\Lambda}_{L}^{6-\left(N_{c}+2\right)} \operatorname{det}\left(\mu^{-1} \widehat{M}\right)}{q^{N_{f}+2} \widetilde{q}^{N_{f}+2}}=-\frac{\operatorname{det} \widehat{M}}{\Lambda_{L}^{3 N_{c}-\left(N_{c}+1\right)}},
$$

where use was made of (5.6). Adding this to the superpotential (5.15) and dropping the hats and the $L$ subscript, the superpotential of the low energy theory with $N_{f}=N_{c}+1$ light flavors is

$$
W=\frac{1}{\Lambda^{2 N_{c}-1}}\left(M_{\tilde{j}}^{i} B_{i} \widetilde{B}^{\tilde{j}}-\operatorname{det} M\right) .
$$

This is precisely the superpotential (4.20). In the electric description (4.20) is associated with strong coupling effects. In the magnetic description it is thus rederived in a weakly coupled framework.

Another way to analyze the theory with mass terms is to consider the massless theory for generic values of $\langle M\rangle$. The dual quarks acquire mass $\mu^{-1} M$ and the low energy magnetic theory is pure glue $S U\left(N_{f}-N_{c}\right)$ Yang-Mills theory with scale $\widetilde{\Lambda}_{L}^{3\left(N_{f}-N_{c}\right)}=\mu^{-N_{f}} \widetilde{\Lambda}^{3\left(N_{f}-N_{c}\right)-N_{f}} \operatorname{det} M$. As in (4.10), gluino condensation in this theory leads to an effective superpotential

$$
W_{\mathrm{eff}}=\left(N_{f}-N_{c}\right) \widetilde{\Lambda}_{L}^{3}=\left(N_{c}-N_{f}\right)\left(\frac{\Lambda^{3 N_{c}-N_{f}}}{\operatorname{det} M}\right)^{1 /\left(N_{c}-N_{f}\right)}
$$


where we used (5.6) (the $(-1)^{N_{f}-N_{c}}$ sign in that relation plays a crucial role in getting the correct overall sign here), which is the same as the continuation of (4.6) to these values of $N_{c}, N_{f}$. This guarantees that the superpotentials (4.6) and the expectation values of $\left\langle M_{\tilde{j}}^{i}\right\rangle$ are reproduced correctly when mass terms are added to the magnetic theory.

The infrared fixed point can also be deformed by giving the fields in the electric theory expectation values along the $D$-flat directions (3.3). Consider, for example, large $\left\langle Q^{N_{f}}\right\rangle=\left\langle\widetilde{Q}_{\tilde{N}_{f}}\right\rangle$, breaking the electric $S U\left(N_{c}\right)$ theory with $N_{f}$ flavors to $S U\left(N_{c}-1\right)$ with $N_{f}-1$ light flavors. The low energy electric theory has scale $\Lambda_{L}^{3\left(N_{c}-1\right)-\left(N_{f}-1\right)}=\Lambda^{3 N_{c}-N_{f}} /\left\langle Q^{N_{f}} \widetilde{Q}_{N_{f}}\right\rangle$ and the fixed point is at weaker coupling. In the magnetic description, the large $\left\langle M_{\tilde{N}_{f}}^{N_{f}}\right\rangle$ gives a large mass $\mu^{-1}\left\langle M_{\tilde{N}_{f}}^{N_{f}}\right\rangle$ to the flavor $q_{N_{f}} \widetilde{q}^{\tilde{N}_{f}}$. The low energy magnetic theory is $S U\left(N_{f}-N_{c}\right)$ with $N_{f}-1$ light flavors and scale $\widetilde{\Lambda}_{L}^{3\left(N_{f}-N_{c}\right)-\left(N_{f}-1\right)}=\mu^{-1}\left\langle M_{\tilde{N}_{f}}^{N_{f}}\right\rangle \widetilde{\Lambda}^{3\left(N_{c}-N_{f}\right)-N_{f}}$. The low energy magnetic theory is at stronger coupling and is the dual of the low energy electric theory. Note that the flow preserves (5.6) and (5.10). Deformations along the flat directions with $\langle B\rangle \neq 0$ directions were analyzed in [39].

Classically, and to all orders of perturbation theory, the electric and magnetic theories have different moduli spaces of vacua - it is only after taking nonperturbative effects into account that they are seen to be identical. For example, in the electric theory there is a classical constraint $\operatorname{rank}\langle M\rangle \leq N_{c}$. In the dual theory, $M$ is an independent field whose expectation value is unconstrained to all orders of perturbation theory - the constraint arises in the dual theory by quantum effects! Defining $N_{i}^{\tilde{j}}=q_{i} \widetilde{q}^{\tilde{j}}$, the $M$ equations of motion imply that vacua of $(5.5)$ are at $\left\langle N_{i}^{\tilde{j}}\right\rangle=0$. However, because the magnetic theory has $N_{f}-\operatorname{rank}\langle M\rangle$ massless flavors, for $N_{f}-\operatorname{rank}\langle M\rangle\left\langle N_{f}-N_{c}\right.$ the magnetic theory generates a superpotential analogous to (4.6) and there is no vacuum with $\langle N\rangle=0$. The vacua of the dual theory thus also satisfy $\operatorname{rank}\langle M\rangle \leq N_{c}$ but as a result of quantum effects rather than as a classical constraint. Similarly, for $\operatorname{rank}\langle M\rangle=N_{c}$, the magnetic theory has $N_{f}-N_{c}=\widetilde{N}_{c}$ massless fields and thus develops a constraint analogous to (4.15)

$$
\operatorname{det} N-b \widetilde{b}=\widetilde{\Lambda}_{L}^{2 \widetilde{N}_{c}}
$$

where $\widetilde{\Lambda}_{L}^{2} \widetilde{N}_{c}=\operatorname{det}^{\prime}\left\langle\mu^{-1} M\right\rangle \widetilde{\Lambda}^{3} \widetilde{N}_{c}-N_{f}$, with $\operatorname{det}^{\prime}\left\langle\mu^{-1} M\right\rangle$ the product of the $N_{c}$ nonzero eigenvalues of $\left\langle\mu^{-1} M\right\rangle$. Using the $M$ equation of motion, $\langle N\rangle=0$, and the mapping (5.10) and (5.6), the relation (5.19) yields

$$
\langle B \widetilde{B}\rangle=\operatorname{det}^{\prime}\langle M\rangle \text {. }
$$

This derives a constraint which is classical in the electric description (it was discussed following (3.3)) via quantum dynamics in the dual theory. In addition to 
providing a necessary check on the duality, the fact that classical relations arise via quantum effects in the dual is interesting in that, conversely, quantum effects in one theory can be related to classical identities in the dual.

\section{6. $S O\left(N_{c}\right)$ with $N_{f} \times \mathbf{N}_{\mathbf{c}}$}

In the $S U\left(N_{c}\right)$ theories discussed above, with matter in the fundamental representation, there is no invariant distinction between Higgs and confinement [2]. This is not the case in theories based on $S O\left(N_{c}\right)$ with $N_{f} \times \mathbf{N}_{\mathbf{c}}$ because the matter cannot screen sources in the spinor representation 0 . These theories, therefore, lead to a clearer picture of the dynamics. In particular, here the transition from the Higgs phase to the Confining phase occurs with a well defined phase transition.

Many of the results in these $S O\left(N_{c}\right)$ theories [29,21, 40,41, 8, 42] are similar to the results in $S U\left(N_{c}\right)$, showing that some phenomena are generic. These $S O\left(N_{c}\right)$ theories also exhibit many new phenomena, which are not present in the $S U\left(N_{c}\right)$ examples. The most dramatic of them is oblique confinement [43,44], driven by the condensation of dyons (particles with both electric and magnetic charges). This phenomenon is best described by another equivalent theory - a dyonic theory. Therefore, these theories exhibit electric-magnetic-dyonic triality [42,45]. The discussion here will be brief; many more details can be found in [42].

\subsection{The phases}

For $N_{f} \geq 3\left(N_{c}-2\right)$ massless quarks $Q$ in the $N_{c}$ dimensional representation of $S O\left(N_{c}\right)$, the theory is not asymptotically free and the infrared theory is thus in a free electric phase. For $\frac{3}{2}\left(N_{c}-2\right)<N_{f}<3\left(N_{c}-2\right)$, the theory is asymptotically free and flows to a non-Abelian Coulomb phase fixed point in the infrared. This phase has a dual magnetic description in terms of an $S O\left(N_{f}-N_{c}+4\right)$ gauge theory which we review below. For $N_{c}-2<N_{f} \leq \frac{3}{2}\left(N_{c}-2\right)$, the theory is in a free magnetic phase with a dynamically generated composite $S O\left(N_{f}-N_{c}+4\right)$ gauge invariance. For $N_{f}=N_{c}-2$, the theory is in an Abelian Coulomb phase.

\subsection{Duality}

As discussed in [8,42], the infra-red behavior of these theories has a dual, magnetic description in terms of an $S O\left(N_{f}-N_{c}+4\right)$ gauge theory with $N_{f}$ flavors of dual quarks $q_{i}$ and an additional gauge singlet field $M^{i j}=Q^{i} \cdot Q^{j}$ with a superpotential

$$
W=\frac{1}{2 \mu} M^{i j} q_{i} \cdot q_{j}
$$

5 We will limit our considerations to the Lie algebra and not distinguish between $S O\left(N_{c}\right)$ and $\operatorname{Spin}\left(N_{c}\right)$ 
(an additional term is required for $N_{f}=N_{c}-1$ ). The scale $\mu$ is needed for the same reason as in the $S U\left(N_{c}\right)$ duality. For generic $N_{c}$ and $N_{f}$ the scale $\widetilde{\Lambda}$ of the magnetic theory is related to the scale $\Lambda$ of the electric theory by

$$
2^{8} \Lambda^{3\left(N_{c}-2\right)-N_{f}} \widetilde{\Lambda}^{3\left(N_{f}-N_{c}+2\right)-N_{f}}=(-1)^{N_{f}-N_{c}} \mu^{N_{f}} .
$$

The constant $2^{8}$ reflects the $\overline{D R}$ conventions 6 .

The electric theory has an anomaly free global $S U\left(N_{f}\right) \times U(1)_{R}$ symmetry with the fields $Q$ transforming as $\left(N_{f}\right)_{\left(N_{f}-N_{c}+2\right) / N_{f}}$. The dual theory has the same global symmetry with $M$ transforming as $Q \cdot Q$ and $q$ transforming as $\left(\bar{N}_{f}\right)_{\left(N_{c}-2\right) / N_{f}}$, which is anomaly free and respected by the superpotential (6.1). Furthermore, the 'tHooft anomalies of this magnetic spectrum match those of the electric spectrum.

For $N_{c}-2<N_{f} \leq \frac{3}{2}\left(N_{c}-2\right)$, the magnetic degrees of freedom are free in the infra-red while, for $\frac{3}{2}\left(N_{c}-2\right)<N_{f}<3\left(N_{c}-2\right)$, the electric and the magnetic theories flow to the same non-trivial fixed point of the renormalization group. Although the two theories are different away from the extreme infra-red, they are completely equivalent at long distance. This means that the two (super) conformal field theories at long distance are identical, having the same correlation functions of all of the operators, including high dimension (irrelevant) operators.

The gauge invariant (primary) chiral operators of the electric theory are

$$
\begin{aligned}
M^{i j} & =Q^{i} Q^{j} \\
B^{\left[i_{1}, \ldots, i_{N_{c}}\right]} & =Q^{i_{1}} \ldots Q^{i_{N_{c}}} \\
b^{\left[i_{1}, \ldots, i_{N_{c}-4}\right]} & =W_{\alpha}^{2} Q^{i_{1}} \ldots Q^{i_{N_{c}-4}} \\
\mathcal{W}_{\alpha}^{\left[i_{1}, \ldots, i_{N_{c}-2}\right]} & =W_{\alpha} Q^{i_{1}} \ldots Q^{i_{N_{c}-2}}
\end{aligned}
$$

with the gauge indices implicit and contracted. These operators get mapped to gauge invariant operators of the magnetic theory as

$$
\begin{aligned}
M^{i j} & \rightarrow M^{i j} \\
B^{\left[i_{1} \ldots i_{N_{c}}\right]} & \rightarrow \epsilon^{i_{1} \ldots i_{N_{f}}} \widetilde{b}_{i_{N_{c}+1} \ldots i_{N_{f}}} \\
b^{\left[i_{1} \ldots i_{N_{c}-4}\right]} & \rightarrow \epsilon^{i_{1} \ldots i_{N_{f}}} \widetilde{B}_{\left[i_{N_{c}-3} \ldots i_{N_{f}}\right]} \\
\mathcal{W}_{\alpha}^{\left[i_{1}, \ldots, i_{N_{c}-2}\right]} & \rightarrow \epsilon^{i_{1} \ldots i_{N_{f}}}\left(\widetilde{\mathcal{W}}_{\alpha}\right)_{\left[i_{N_{c}-1}, \ldots, i_{N_{f}}\right]},
\end{aligned}
$$

6 In these conventions the matching relation between the scale of the high energy theory with $N_{f}$ flavors and the mass term $W_{\text {tree }}=\frac{1}{2} m Q^{N_{f}} \cdot Q^{N_{f}}$ and the low energy theory with $N_{f}-1$ flavors is $\Lambda_{L}^{3\left(N_{c}-2\right)-N_{f}+1}=m \Lambda^{3\left(N_{c}-2\right)-N_{f}}$ (it is $\Lambda_{L}^{6-2\left(N_{f}-1\right)}=m^{2} \Lambda^{6-2 N_{f}}$ for $\left.N_{c}=3\right)$. Similarly, the matching relation associated with breaking $S O\left(N_{c}\right)$ with $N_{f}$ vectors to $S O\left(N_{c}-1\right)$ with $N_{f}-1$ vectors by an expectation value $\left\langle Q^{N_{f}}\right\rangle$ is $\Lambda_{L}^{3\left(N_{c}-2\right)-N_{f}-2}=\Lambda^{3\left(N_{c}-2\right)-N_{f}}\left(M^{N_{f} N_{f}}\right)^{-1}$ (for breaking $S O(4) \cong S U(2)_{1} \times S U(2)_{2}$ to $S O(3)$, it is $\left.\Lambda_{L}^{6-2\left(N_{f}-1\right)}=4 \Lambda_{1}^{6-N_{f}} \Lambda_{2}^{6-N_{f}}\left(M^{N_{f} N_{f}}\right)^{-2}\right)$. 
where $\widetilde{B}, \widetilde{b}$, and $\widetilde{\mathcal{W}}_{\alpha}$ are the magnetic analogs of the operators in (6.3). These maps are compatible with the global symmetries discussed above.

Deforming the electric theory along a flat direction of non-zero $\left\langle Q^{N_{f}}\right\rangle$ gives $S O\left(N_{c}-1\right)$ with $N_{f}-1$ massless flavors and the new fixed point is at weaker coupling. In the dual description this deformation gives a mass $\mu^{-1}\left\langle M^{N_{f} N_{f}}\right\rangle$ to $q_{N_{f}}$ and the low energy dual theory is $S O\left(N_{f}-N_{c}+4\right)$ with $N_{f}-1$ massless quark flavors, which is the dual of the low energy electric theory. In the magnetic description the new fixed point is at stronger coupling and the relation (6.2) is preserved in the low energy theories.

Some classical identities of the electric theory arise from quantum effects in the dual. For example, it is seen classically in the electric theory that $\operatorname{rank}(M) \leq$ $N_{c}$. In the magnetic description, this condition arises because, for larger rank, a superpotential is dynamically generated and the magnetic theory has no vacuum. Similarly, the electric theory has a classical relation that, when $\operatorname{rank}(M)=N_{c}$, the baryon $B^{\left[i_{1}, \ldots i_{N_{c}}\right]}$ has the non-zero eigenvalue $B= \pm \sqrt{\operatorname{det}^{\prime} M}$. This is mapped under duality to the relation that, for $N_{f}=N_{c}-4$, the operator $b$ defined above is given by $b= \pm \sqrt{\Lambda^{2\left(N_{c}-1\right)}}$, which is related to gaugino condensation.

Deforming the electric theory by giving a mass to $Q^{N_{f}}$ gives $S O\left(N_{c}\right)$ with $N_{f}-$ 1 massless flavors and the new fixed point is at stronger coupling. In the dual description, adding $W_{\text {tree }}=\frac{1}{2} m M^{N_{f} N_{f}}$ to (6.1) and integrating out the massive fields gives $\left\langle q_{N_{f}} \cdot q_{N_{f}}\right\rangle=-\mu m$ which, along with the $D$-terms, implies that the dual gauge group is broken by the Higgs mechanism to $S O\left(N_{f}-N_{c}+3\right)$ with $N_{f}-1$ massless flavors, which is the dual of the low energy electric theory. In the magnetic description the new fixed point is at weaker coupling and the relation (6.2) is preserved in the low energy theories.

For $N_{f}=N_{c}-1$ the dual gauge group is $S O(3)$ and the superpotential (6.1) is modified to

$$
W=\frac{1}{2 \mu} M^{i j} q_{i} \cdot q_{i}-\frac{1}{64 \Lambda_{N_{c}, N_{c}-1}^{2 N_{c}-5}} \operatorname{det} M
$$

Also, the relation (6.2) between the scales is modified in this case to

$$
2^{14}\left(\Lambda_{N_{c}, N_{c}-1}^{2 N_{c}-5}\right)^{2} \widetilde{\Lambda}_{3, N_{c}-1}^{6-2\left(N_{c}-1\right)}=\mu^{2\left(N_{c}-1\right)}
$$

These modifications arise upon going from $N_{f}=N_{c}$ with a mass term added for $Q^{N_{c}}$ to the low energy theory with $N_{f}=N_{c}-1$ because of peculiarities associated with the breaking of the magnetic $S O(4) \cong S U(2) \times S U(2)$ to the diagonally embedded magnetic $S O(3)$ [8,42. 


\section{Abelian Coulomb phase}

\subsection{General features}

Consider a general theory with a low energy $N=1$ Abelian Coulomb phase. For simplicity we consider only the situation with a single photon. As discussed in sect. 2 , the effective gauge coupling $\tau_{\text {eff }}\left(X_{r}, g_{I}\right)$ depends holomorphically on the light fields $X_{r}$ and the coupling constants, including the scale $\Lambda$ of the underlying nonAbelian $G$ theory in which the low energy, Abelian theory is embedded. However, $\tau_{\text {eff }}$ is not a single valued function of $X_{r}$ and the couplings. This is possible because $\tau$ gives a redundant description of the physics: $\tau$ is physically identified under $S L(2, Z)$ transformations, generated by $S: \tau \rightarrow-1 / \tau$, which is associated with the possibility of exchanging electric with magnetic in ordinary Maxwell theory, and $T: \tau \rightarrow \tau+1$, which is a unit shift of the theta angle. In order for physics to be single valued, $\tau$ need only be a section of an $S L(2, Z)$ bundle [40].

For simplicity, we will consider the case where $\tau$ only depends on a single light field (or a single function of the light fields) $U$ whose expectation value serves as an order parameter for breaking the underlying non-Abelian $G$ gauge theory to a $U(1)$ subgroup. For large $U$, the $G$ gauge theory is weakly coupled and the one loop beta function in the microscopic theory leads to

$$
\tau \approx \frac{i b}{2 \pi} \log \frac{U}{\Lambda^{p}}
$$

for some integers $b$ and $p$, where $\Lambda$ is the scale of $G$. As we circle around infinity, $U \rightarrow e^{2 \pi i} U, \tau \rightarrow \tau-b$; i.e. $\tau$ is transformed by $\mathcal{M}_{\infty}=T^{-b}$. So even at weak coupling $\tau$ is not single valued. The low energy effective gauge coupling $\frac{1}{g_{\text {eff }}^{2}} \sim \operatorname{Im} \tau$ is invariant under $\mathcal{M}_{\infty}$. However, if $\operatorname{Im} \tau$ were single valued everywhere in the interior of the moduli space, because it is a harmonic function, it couldn't be everywhere positive definite [40]. There would then be regions in the moduli space where $g_{\text {eff }}$ is imaginary. This unphysical conclusion can be avoided if the topology of the moduli space is complicated in the interior or, as found in [40], there are several (at least two) singular values $U_{i}$ of $U$ with monodromies $\mathcal{M}_{i}$ around them which do not commute with $\mathcal{M}_{\infty}=T^{-b}$.

The monodromies $\mathcal{M}_{i}$ around the $U_{i}$ must have a physical interpretation. The simplest one is that they are associated with $k_{i}$ massless particles at the singularity. The low energy superpotential near $U_{i}$ then has the form

$$
W_{L}^{(i)}=\left(U-U_{i}\right) \sum_{l=1}^{k_{i}} c_{l}^{(i)} \widetilde{E}_{l}^{(i)} E_{l}^{(i)}+\mathcal{O}\left(\left(U-U_{i}\right)^{2}\right)
$$


where $\widetilde{E}_{l}^{(i)}$ and $E_{l}^{(i)}$ are the new massless states. If the constants $c_{l}^{(i)}$ are nonzero, these states acquire a mass of order $\mathcal{O}\left(\left(U-U_{i}\right)\right)$ away from the singularity. Therefore, the one loop beta function in the low energy theory leads to

$$
\tau_{i} \approx-\frac{i k_{i}}{2 \pi} \log \left(U-U_{i}\right)
$$

(we assume for simplicity that, as in [40,5], all the $E_{l}^{(i)}$ have charge one; the generalization to other cases is straightforward) where $\tau_{i}$ is the coupling to the low energy photon. $\tau_{i}$ is related to $\tau$ in the asymptotic region by a duality transformation $N_{i}$. It is clear from (7.3) that the monodromy in $\tau_{i}$ is $T^{k_{i}}$. Therefore, the monodromy in $\tau$ is

$$
\mathcal{M}_{i}=N_{i}^{-1} T^{k_{i}} N_{i}
$$

For $\mathcal{M}_{i}$ to not commute with $\mathcal{M}_{\infty}=T^{-b}$, the transformation $N_{i}$ must include $S$. This means that the massless particles $E_{l}^{(i)}$ at $U_{i}$ are magnetically charged.

As discussed in [40], because $\tau$ is a section of an $S L(2, \mathbf{Z})$ bundle it is naturally described as the modular parameter $\tau$ of a torus. A torus is conveniently described by the one complex dimensional curve in $C^{2}$ :

$$
y^{2}=x^{3}+A x^{2}+B x+C,
$$

where $(x, y) \in C^{2}$ and $A, B$ and $C$ are parameters. The modular parameter of the torus (7.5) is given by

$$
\tau(A, B, C)=\frac{\int_{b} \frac{d x}{y}}{\int_{a} \frac{d x}{y}},
$$

where $a$ and $b$ refer to a basis of cycles around the branch cuts of (7.5) in the $x$ plane. The problem of finding the section $\tau$ is thus reduced to the simpler problem of finding $A, B$, and $C$ as functions (rather than sections) of $U$ and the various coupling constants and scales.

The $\tau$ obtained from (7.5) is singular when the torus is singular, which is when

$$
x^{3}+A x^{2}+B x+C=0 \quad \text { and } \quad 3 x^{2}+2 A x+B=0 .
$$

Eliminating $x$, this is when the discriminant of the cubic equation in (7.7) vanishes: $\Delta(A, B, C)=0$ where

$$
\Delta=4 A^{3} C-B^{2} A^{2}-18 A B C+4 B^{3}+27 C^{2} .
$$

As discussed in [40.5], the order of the zero can be used to determine the monodromy (7.4) around the singularity and, thus, the charge of the associated massless fields. 
Important constraints [5] on the dependence of the coefficients in (7.5) on $U$ and the coupling constants are the following:

1. In the weak coupling limit $\Lambda=0$ the curve should be singular for every $U$. Without loss of generality we can then take $y_{0}^{2}=x^{2}(x-U)$.

2. The parameters $A, B, C$ in (7.5) are holomorphic in $U$ and the various coupling constants. This guarantees that $\tau$ is holomorphic in them.

3. The curve (7.5) must be compatible with all the global symmetries of the theory including those which are explicitly broken by the coupling constants or the anomaly.

4. In various limits (e.g. as some mass goes to zero or infinity) we should recover the curves of other models.

5. The curve should have physical monodromies around the singular points.

\section{2. $S O\left(N_{c}\right)$ with $N_{f}=N_{c}-2$}

For $N_{f}=N_{c}-2$ the gauge group is broken by $\langle Q\rangle$ to $S O(2) \cong U(1)$ and the theory has an Abelian Coulomb phase. Applying the considerations of the previous section, it is found that the Abelian Coulomb phase has an effective gauge coupling $\tau_{\text {eff }}(\operatorname{det} M, \Lambda)$ which is exactly given by the curve 42

$$
y^{2}=x^{3}+x^{2}\left(-\operatorname{det} M+8 \Lambda^{2 N_{c}-4}\right)+16 \Lambda^{4 N_{c}-8} x
$$

For example, at weak coupling (large $\operatorname{det}\langle M\rangle)$, (7.9) properly reproduces the one loop beta function of $S O\left(N_{c}\right)$ with $N_{f}$ fields $Q$. The curve $(7.9)$ has singularities at the solutions of $(7.8)$, which are $\operatorname{det} M=0$ and $\operatorname{det} M=16 \Lambda^{2 N_{c}-4}$.

Classically, the submanifold $\operatorname{det} M=0$ has a singularity associated with a nonAbelian Coulomb phase with some of the $S O\left(N_{c}\right)$ gluons becoming massless. In the quantum theory, the monodromy of $\tau$ implied by $(7.9)$ around $\operatorname{det} M=0$ reveals that this submanifold of the moduli space of vacua is actually in an Abelian free magnetic phase associated with massless monopoles. At the origin there are $N_{f}$ massless monopoles $q_{i}^{ \pm}$. Away from the origin, they obtain a mass matrix proportional to $\langle M\rangle$ via

$$
W \sim M^{i j} q_{i}^{+} q_{j}^{-}
$$

The monodromy obtained from $(7.9)$ around $\operatorname{det} M=16 \Lambda^{2 N_{c}-4}$ reveals that this submanifold is in a free dyonic phase associated with a single dyonically charged field $E$ which is massless at $\operatorname{det} M=16 \Lambda^{2 N_{c}-4}$; near the singularity the dyon gets a mass via

$$
W \sim\left(\operatorname{det} M-16 \Lambda^{2 N_{c}-4}\right) E^{+} E^{-}
$$


The quantum numbers of the dyon $E$ are such that $E \sim q_{i} Q^{i}$. For example, if we take the $q_{i}$ to be monopoles with zero electric charge, $E$ is a dyon with electric charge one. Thus, as a matter of convention, we will refer to the $q_{i}$ as monopoles and to $E$ as a dyon.

To summarize, we should expect to find two dual descriptions of the original electric theory, one in which the monopoles $q_{i}$ are taken as fundamental fields and one in which the dyon $E$ is a taken as a fundamental field.

Consider the duality discussed in the previous section. For $N_{f}=N_{c}-2$ the dual magnetic theory is $S O(2) \cong U(1)$ with $N_{f}$ charged fields $q_{i}^{ \pm}$and neutral fields $M^{i j}$ with a superpotential

$$
W \sim M^{i j} q_{i}^{+} q_{j}^{-} .
$$

To see the relation of this Abelian dual to the non-Abelian duals considered in the previous section, consider flowing from $N_{f}=N_{c}-1$ to $N_{f}=N_{c}-2$ by giving an electric quark a mass. In the magnetic theory this generically Higgses the magnetic $S O(3)$ to a magnetic $S O(2)$ and the low energy superpotential is (7.12). (There are additional contributions to (7.12) from instantons in the broken magnetic $S O(3)$ [42].) The components $q_{i}^{ \pm}$of the magnetic quarks become magnetic monopoles in the low energy Abelian Coulomb phase. The $N_{f}$ monopoles $q_{i}^{ \pm}$with superpotential (7.12) is precisely the situation (7.10), determined above from the curve (7.9).

The dyon (7.11) is seen by a strong coupling analysis in either the electric or the magnetic theories [8,42]. There is also a dyonic dual description in which the dyons $E$ and $\widetilde{E}$ are taken as fundamental fields. Just as the $N_{f}=N_{c}-2$, Abelian magnetic dual can be obtained by flowing down from the non-Abelian, $N_{f}=N_{c}-1, S U(2)$ magnetic dual, the Abelian dyonic dual can be obtained by flowing down from a dyonic dual, $N_{f}=N_{c}-1, S O\left(N_{c}\right)$ gauge theory. This dyonic dual theory [42] has composite $S O\left(N_{c}\right)$ gauge fields interacting with a theta angle which differs from that of the original electric theory by $\pi$ and it has composite quarks $d^{i}$, satisfying $d^{i} \cdot d^{j}=M^{i j}$, with superpotential

$$
W=-\frac{1}{32 \Lambda^{2 N_{c}-5}} \operatorname{det} d \cdot d .
$$

Adding $W_{\text {tree }}=\frac{1}{2} m Q^{N_{c}-1} \cdot Q^{N_{c}-1}=\frac{1}{2} m d^{N_{c}-1} \cdot d^{N_{c}-1}$ the low energy electric theory has $N_{f}=N_{c}-2$ and the low energy dyonic dual has an unbroken $S O(2)$ with a charged pair which becomes massless at $\operatorname{det} M=16 \Lambda_{L}^{2 N_{c}-4}$, coming from $d^{N_{c}-1}$, which is seen at weak coupling.

Consider giving a mass to the $N_{c}-2$-th flavor by adding $W_{\text {tree }}=\frac{1}{2} m M^{N_{c}-2, N_{c}-2}$, giving $S O\left(N_{c}\right)$ with $N_{f}=N_{c}-3$ light flavors at low energy. Adding $W_{\text {tree }}$ eliminates the Coulomb phase and the low energy theory has two distinct branches. One branch is associated with adding $W_{\text {tree }}$ to (7.12) which, upon integrating out the massive 
fields, gives the monopole condensate $\left\langle q_{N_{f}} q_{N_{f}}\right\rangle \sim m$, yielding a confining phase by the dual Meissner effect. The low energy theory has a moduli space of vacua labeled by $\langle\widehat{M}\rangle$ with $N_{c}-3$ massless fields at the origin, with a superpotential given by

$$
W \sim M^{i j} q_{i} q_{j}
$$

where we dropped the hats and $i, j=1 \ldots N_{c}-3$. In the magnetic description, the fields $q_{i}$ are left-over components of the magnetic quarks. By the map (6.4), the quark component $q_{i}$ in this case is mapped to the exotic $q_{i} \sim(Q)^{N_{c}-4} W_{\alpha} W^{\alpha}$. Intuitively, one thinks of such exotics as being large and heavy bound states. Here we see that they become massless at $\langle M\rangle=0$. This phenomenon is similar to the massless composite mesons and baryons found in $S U\left(N_{c}\right)$ with $N_{f}=N_{c}+1$ [33]. As was the case there, we have confinement without chiral symmetry breaking.

The other branch is associated with adding $W_{\text {tree }}$ to (7.11). Integrating out the massive fields gives the dyon condensate $\left\langle E^{+} E^{-}\right\rangle \sim m / \operatorname{det} \widehat{M}$, yielding an oblique confining phase. The low energy theory has a superpotential

$$
W_{\text {oblique }}=\frac{8 \Lambda_{L}^{2 N_{c}-3}}{\operatorname{det} \widehat{M}} .
$$

In the electric description, this superpotential is associated with gaugino condensation in the unbroken electric $S O(3)$ along with contributions from instantons in the broken $S O\left(N_{c}\right) / S O(3)$ [42]. In the magnetic description (7.15) arises from dyon condensation. In the dyonic description (7.13) it arises at tree level.

\section{8. $S O(3) \cong S U(2)$ examples}

The discussion of the previous sections is modified slightly for $S O(3) \cong S U(2)$, where the 3 dimensional representation is the adjoint representation.

\subsection{One adjoint, Q; an Abelian Coulomb phase}

This is the $N=2$ theory discussed in [40]. The theory has a quantum moduli space of vacua labeled by the expectation value of the massless meson field $M=Q^{2}$. The $S U(2)$ gauge symmetry is broken to $U(1)$ on this moduli space, so the theory has a Coulomb phase with a massless photon.

As discussed in 40,50, the effective gauge coupling in the Coulomb phase is given by the $\tau_{\text {eff }}(M)$ obtained from the curve

$$
y^{2}=x^{3}-M x^{2}+4 \Lambda^{4} x .
$$

(This curve is expressed using the convention for the normalization of $\tau$ discussed in [5], $\tau=\frac{\theta}{\pi}+8 \pi i g^{-2}$.) This gives a $\tau_{\mathrm{eff}}(M)$ which has singularities associated with 
a massless magnetic monopole field $q_{(+)}$, at $M=4 \Lambda^{2}$ and a massless dyon $q_{(-)}$at $M=-4 \Lambda^{2}$. Therefore, these two points are in a free magnetic and a free dyonic phase, respectively. Here $q_{(+)}$is a doublet charged under the magnetic $U(1)_{M}$, which is related to the electric $U(1)_{E}$ by the electric-magnetic transformation $S$ : $F \rightarrow \widetilde{F}$ (modulo $\Gamma(4) \subset S L(2, Z)$ ). Similarly, $q_{(-)}$is a doublet charged under a dyonic $U(1)_{D}$, related to $U(1)_{E}$ by the $S L(2, Z)$ transformation $S T^{2}$ (again, modulo $\Gamma(4) \subset S L(2, Z))$, where $T$ is a rotation of the theta angle by $\pi$. Near where these fields are massless, they couple through the effective superpotentials

$$
W_{ \pm} \sim\left(M \mp 4 \Lambda^{2}\right) q_{( \pm)} \cdot q_{( \pm)} .
$$

Referring to the underlying $S U(2)$ theory as "electric," we can say that it has two dual theories. One of them, which we can refer to as the "magnetic dual," describes the physics around $M=4 \Lambda^{2}$ with the superpotential $W_{+}$. The other dual, which can be called the "dyonic dual," is valid around $M=-4 \Lambda^{2}$ and is described by $W_{-}$.

Consider giving $Q$ a mass by adding a term $W_{\text {tree }}=\frac{1}{2} m M$ in the electric theory. Adding $W_{\text {tree }}$ to (8.2), the equations of motion give $\left\langle q_{( \pm)} \cdot q_{( \pm)}\right\rangle \sim m$ and lock $\langle M\rangle= \pm 4 \Lambda^{2}$. The condensate of monopoles/dyons Higgses the dual theory and thus gives confinement/oblique confinement of the electric theory by the dual Meissner effect 40].

Consider analyzing, as in sect. 2.3, the 1PI effective action for this theory with sources. Starting from the analog of (4.10) for this theory, $W_{L}(m)= \pm 2\left(\Lambda^{4} m^{2}\right)^{1 / 2}$, equations (2.8) and (2.9) give $W=0$ with the constraint $\langle M\rangle= \pm 4 \Lambda^{2}$. Indeed, adding the source $m$ for $M$ drives the theory to the confining or oblique confining phase with $\langle M\rangle= \pm 4 \Lambda^{2}$. The Coulomb phase cannot be explored in the theory with a mass term for $Q$. As discussed in sect. 2.3, this method of analyzing the theory must fail to capture some of the physics because the theory without sources has massless fields (the monopole or dyon) which cannot be represented by the gauge invariant observables.

Because this theory actually has $N=2$ supersymmetry, it can be further analyzed using the additional techniques applicable for $N=2$ theories, yielding the Kahler potential for $Q$ and a BPS mass bound [40].

\section{2. $S U(2)$ with two adjoints; A non-Abelian Coulomb phase}

This theory has $N=1$ (not $N=2$ ) supersymmetry. Writing the matter fields as $Q^{i}$ with $i=1,2$ a flavor index, there is a 3 complex dimensional moduli space of

7 We use the conventions of [21, 30] where the normalization of $\Lambda^{2}$ (in the $\overline{D R}$ scheme) differs by a factor of 2 from that of [40]; our order parameter $M$ is related to $u$ of [40] as $u=\frac{1}{2} M$. 
classical vacua parameterized by the expectation values of the gauge singlet fields $M^{i j}=Q^{i} \cdot Q^{i}$. In the generic vacuum $\left\langle Q^{1}\right\rangle$ breaks $S U(2)$ to a $U(1)$ which is then broken by $\left\langle Q^{2}\right\rangle$. For $\operatorname{det}\left\langle M^{i j}\right\rangle \neq 0$, the gauge group is completely broken and the theory is in the Higgs phase. On the non-compact two complex dimensional subspace of vacua with $\operatorname{det} M=0$, there is an unbroken $U(1)$ gauge symmetry and thus a light photon along with a pair of massless electrically charged fields. At the point $\langle M\rangle=0$ the $S U(2)$ gauge group is unbroken.

We now turn to the quantum theory. The theory has the global symmetry group $S U(2) \times U(1)_{R}$, with $Q$ transforming as $\mathbf{2}_{\frac{1}{2}}$, which determines that any dynamically generated superpotential must be of the form

$$
W=\frac{c}{\Lambda} \operatorname{det} M
$$

with $c$ a dimensionless constant. Its behavior at $M \rightarrow \infty$ is incompatible with asymptotic freedom, as signaled by the presence of the scale $\Lambda$ in the denominator. Therefore, no superpotential can be generated and the classical vacuum degeneracy outlined above is not lifted quantum mechanically.

The generic ground state with generic $M$ is in the Higgs phase. Consider now the subspace of the moduli space with $\operatorname{det} M=0$. The low energy degrees of freedom there are a single photon, a pair of massless electrically charged fields and some neutral fields. This theory cannot become strong in the infrared. In fact, the loops of the massless charged fields renormalize the electric charge to zero. Therefore, this subspace of the moduli space is in a free electric phase.

Now consider adding a tree level superpotential $W_{\text {tree }}=\frac{1}{2} \operatorname{Tr} m M$. Taking $m=\left(\begin{array}{cc}0 & 0 \\ 0 & m_{2}\end{array}\right), Q^{2}$ gets a mass and can be integrated out. The low energy theory is $S U(2)$ with a single massless adjoint matter field, which is the example of the previous subsection. This low energy theory has a scale $\Lambda_{L}$, which is related to that of the original theory by $\Lambda_{L}^{4}=m_{2}^{2} \Lambda^{2}$, and a massless monopole or dyon at $\left\langle M^{11}\right\rangle= \pm 4 \Lambda_{L}^{2}= \pm 4 m_{2} \Lambda$. Note that as $m_{2} \rightarrow 0$ the point $\langle M\rangle=0$ has both massless monopoles and dyons. These are mutually non-local 8 and signal another phase at this point in the theory with $m_{2}=0$. We interpret this as a non-Abelian Coulomb phase 41].

Starting from the theory with $m_{2} \neq 0$, turning on $m_{1} \neq 0$ drives the monopole or dyon to condense and the vacuum is locked at $\left\langle M^{11}\right\rangle= \pm 4 m_{2} \Lambda$. The + sign is a vacuum with monopole condensation and thus confinement. The - sign is a vacuum with dyon condensation and thus oblique confinement. More generally, these vacua are at $\left\langle M^{i j}\right\rangle= \pm 4 \Lambda \operatorname{det} m\left(m^{-1}\right)^{i j}$. These expectation values can be obtained from

$$
W_{e}=\frac{e}{8 \Lambda} \operatorname{det} M+\frac{1}{2} \operatorname{Tr} m M,
$$

8 A similar situation was found in $N=2 S U(3)$ Yang Mills theory 446]. 
with $e=\mp 1$ for confinement and oblique confinement, respectively.

The theory has various phase branches. For mass $m=0$ there is a Higgs phase which, in terms of $W_{e}$, corresponds to $e=0$. There is a subspace $\operatorname{det} M=0$ in the free electric phase and the point $M=0$ in a non-Abelian Coulomb phase. For $m \neq 0$ but with $\operatorname{det} m=0$ the theory is in the Coulomb phase with a free magnetic point and a free dyonic point. For $\operatorname{det} m \neq 0$ the theory is either confining and described by the superpotential (8.4) with $e=-1$ or it is oblique confining and described by the superpotential (8.4) with $e=1$.

If we consider the 1PI effective action, the analog of (4.10) is $W_{L}(m)=$ $\pm 2\left(\Lambda^{2} \operatorname{det} m\right)^{1 / 2}$. Integrating in gives the confining or oblique confining phase branches of the superpotential (8.4), with $e= \pm 1$, missing the $e=0$ Higgs phase branch. Again, as discussed in sect. 2.3, the 1PI superpotential necessarily fails to capture some of the physics because the theory without the sources has massless particles, the quarks and the gluons, which cannot be represented by the gauge invariant observables.

The analysis of [42] reveals that this electric theory has two dual descriptions, similar to the magnetic and dyonic duals discussed in the previous section, labeled by $\epsilon= \pm 1$. The two dual theories are based on an $S U(2)$ gauge group with two fields $q_{i}$ in its adjoint representation and three gauge singlet fields $M^{i j}$. The difference between the two theories is in the superpotential

$$
W_{\epsilon}=\frac{1}{12 \sqrt{\Lambda \widetilde{\Lambda}}} M^{i j} q_{i} \cdot q_{j}+\epsilon\left(\frac{1}{24 \Lambda} \operatorname{det} M+\frac{1}{24 \widetilde{\Lambda}} \operatorname{det} q_{i} \cdot q_{j}\right),
$$

where $\widetilde{\Lambda}$ is the scale of the dual $S U(2)$ (we expressed $\mu$ in terms of $\Lambda$ and $\widetilde{\Lambda}$ ). The theory with $\epsilon=1$ is a "magnetic" dual and that with $\epsilon=-1$ a "dyonic" dual.

We now analyze the dynamics of these dual theories. Since they are similar to the original electric theory, we proceed as we did there. These theories have three phases: Higgs, confining and oblique confining. We study them using the gauge invariant order parameters $N_{i j} \equiv q_{i} \cdot q_{j}$. Its effective superpotential is obtained by writing the tree level superpotential (8.5) in terms of $N$ and adding to it $\frac{\tilde{e}}{8 \Lambda} \operatorname{det} N$ where, in the Higgs, confining and oblique confinement branches, $\tilde{e}=0,-1,1$, respectively

$$
W_{\epsilon, \tilde{e}}=\frac{1}{12 \sqrt{\Lambda \widetilde{\Lambda}}} \operatorname{Tr} M N+\epsilon\left(\frac{1}{24 \Lambda} \operatorname{det} M+\frac{1}{24 \widetilde{\Lambda}} \operatorname{det} N\right)+\frac{\tilde{e}}{8 \widetilde{\Lambda}} \operatorname{det} N .
$$

Now we can integrate out the massive field $N$ to find

$$
W_{\mathrm{eff}}=\frac{1}{8 \Lambda} \frac{\tilde{e}-\epsilon}{1+3 \tilde{e} \epsilon} \operatorname{det} M
$$


This is the same as the effective superpotential (8.4) of the electric theory with

$$
e=\frac{\tilde{e}-\epsilon}{1+3 \tilde{e} \epsilon} .
$$

We see that the various phases are permuted in the different descriptions as:

\section{Theory}

electric

magnetic $(\epsilon=1)$

dyonic $(\epsilon=-1)$

\section{Phases}

$$
\begin{array}{lll}
\text { Higgs }(e=0) & \text { conf. }(e=-1) & \text { obl. conf. }(e=1) \\
\text { obl. conf. }(\tilde{e}=1) & \text { Higgs }(\tilde{e}=0) & \text { conf. }(\tilde{e}=-1) \\
\text { conf. }(\tilde{e}=-1) & \text { obl. conf. }(\tilde{e}=1) & \text { Higgs }(\tilde{e}=0)
\end{array}
$$

It is a simple exercise to check that by dualizing the magnetic and dyonic theories as we above dualized the electric theory (two duals of each), we find permutations of the same three theories. The $S_{3}$ triality permuting the phases and branches is associated with a quotient of the $S L(2, Z)$ electric-magnetic duality symmetry group: the theories are preserved under $\Gamma(2) \subset S L(2, Z)$, leaving the quotient $S_{3}=S L(2, Z) / \Gamma(2)$ with a non-trivial action.

This discussion leads to a new interpretation of the first term in (8.4). In the electric theory this term appears as a consequence of complicated strong coupling dynamics in the confining and the oblique confinement branches of the theory. In the dual descriptions it is already present at tree level.

Consider the theory with a mass $m_{2}$ for $Q^{2}$. As discussed above, the low energy electric theory has a Coulomb phase with massless monopoles or dyons at the strong coupling singularities $\left\langle M^{11}\right\rangle= \pm 4 m_{2} \Lambda$. We now derive this result in the dual theories. Adding $W_{\text {tree }}=\frac{1}{2} m_{2} M^{22}$ to the superpotential (8.5) of the dual theory, the equations of motion give

$$
\begin{aligned}
\frac{1}{12 \sqrt{\Lambda \widetilde{\Lambda}}} q_{2} \cdot q_{2}+\frac{8 \epsilon}{24 \Lambda} M^{11}+\frac{1}{2} m_{2} & =0 & & M^{22}=-\frac{1}{2} \epsilon \sqrt{\frac{\Lambda}{\widetilde{\Lambda}}} q_{1} \cdot q_{1} \\
q_{1} \cdot q_{2} & =0 & M^{12} & =0 .
\end{aligned}
$$

For $q_{2}^{2} \neq 0,\left\langle q_{2}\right\rangle$ breaks the gauge group to $U(1)$ and the remaining charged fields $q_{1}^{ \pm}$couple through the low energy superpotential

$$
\frac{1}{16 \sqrt{\Lambda \widetilde{\Lambda}}}\left(M^{11}-4 \epsilon m_{2} \Lambda\right) q_{1}^{+} q_{1}^{-} .
$$

(This superpotential is corrected by contributions from instantons in the broken magnetic $S U(2)$ theory. However, these are negligible near $M^{11}=4 \epsilon m_{2} \Lambda$.) We 
see that the theory has a charged doublet of massless fields $q_{1}^{ \pm}$at $M^{11}=4 \epsilon m_{2} \Lambda$, exactly as expected from the analysis of the electric theory. There these states appeared as a result of strong coupling effects. Here we see them as weakly coupled states in the dual theories. This is in accord with the interpretation of the $\epsilon=1$ $(\epsilon=-1)$ theory as magnetic (dyonic).

The other monopole point on the moduli space of the theory with $m_{1}=0$ but $m_{2} \neq 0$ is at $M^{11}=-4 \epsilon m_{2} \Lambda$. It arises from strong coupling dynamics in the dual theories. To see that, note that the above analysis is not valid when the expectation value of $q_{2}$ is on the order of or smaller than the mass of $q_{1}$. In that case, $q_{1}$ should be integrated out first. The equations of motion in the low energy theory yield a single massless monopole point at $M^{11}=-4 \epsilon m_{2} \Lambda$ 42].

An analysis similar to the one above leads to a strongly coupled state in the dual theories along the flat directions with $\operatorname{det} M=0$ in the $m=0$ case. This state can be interpreted as the massless quark of the electric theory in that free electric phase.

To conclude, this theory has three branches which are in three different phases: Higgs, confining and oblique confinement (various submanifolds of these branches are in Coulomb, free electric, free magnetic and free dyonic phases). They touch each other at a point in a non-Abelian Coulomb phase. Corresponding to the three branches there are three different Lagrangian descriptions of the theory: electric, magnetic and dyonic. Each of them describes the physics of one of the branches, where it is Higgsed, in weak coupling and the other two in strong coupling.

In both examples of this section, the theory has a discrete symmetry which relates the confining and the oblique confinement phases 9 . Therefore, in these cases the effects of confinement are indistinguishable from the effects of oblique confinement. Correspondingly, the magnetic and the dyonic descriptions are similar - they differ only in the sign of $\epsilon$. In the other $S O\left(N_{c}\right)$ examples discussed in the previous section, these two phases are not related by a symmetry and the two dual descriptions look totally different.

\section{Conclusions}

To conclude, supersymmetric field theories are tractable and many of their observables can be computed exactly 2 . Our analysis led us to find new phases of

9 This symmetry is manifest only in the electric description. In the dual descriptions it is realized as a quantum symmetry [42].

10 Although we did not discuss them here, we would like to point out that many other examples were studied [29, 11, 47, 21, 5, 41, 8, 48, 67] exhibiting many new interesting phenomena. 
non-Abelian gauge theories, like the non-Abelian Coulomb phase with its quantum equivalence and the free magnetic phase with its massless composite gauge fields. The main dynamical lesson is the role of electric-magnetic duality in non-Abelian gauge theories in four dimensions. To be clear we should distinguish several different notions of duality:

1. The exact $\tau \rightarrow-1 / \tau$ duality of Maxwell theory and its generalization to the Montonen-Olive [3] duality of finite, interacting, non-Abelian theories.

2. The $\tau_{\text {eff }} \rightarrow-1 / \tau_{\text {eff }}$ duality of low energy theories with an Abelian Coulomb phase. This is not a symmetry but, rather, an ambiguity in the description of the low energy physics.

3. The duality of two asymptotically free theories which flow to the same nonAbelian Coulomb phase fixed point in the infrared.

4. The duality of the free magnetic phase, which provides a relation between the UV and the IR behavior of a theory which is free in the IR.

There are relations between many of the phenomena discussed above [42,52] but, at a deeper level, they remain to be really understood.

\section{Acknowledgments}

We would like to thank T. Banks, D. Kutasov, R. Leigh, M.R. Plesser, P. Pouliot, S. Shenker, M. Strassler and especially E. Witten for many helpful discussions. This work was supported in part by DOE grant \#DE-FG05-90ER40559. 


\section{References}

[1] N. Seiberg, hep-th/9506077, RU-95-37, IASSNS-HEP-95/46, to appear in the Proc. of PASCOS 95, the Proc. of the Oskar Klein lectures, and in the Proc. of the Yukawa International Seminar '95

[2] L. Susskind, unpublished; T. Banks, E. Rabinovici, Nucl. Phys. B160 (1979) 349; E. Fradkin and S. Shenker, Phys. Rev. D19 (1979) 3682.

[3] C. Montonen and D. Olive, Phys. Lett. 72B (1977) 117; P. Goddard, J. Nuyts and D. Olive, Nucl. Phys. B125 (1977) 1.

[4] H. Osborn, Phys. Lett. 83B (1979) 321; A. Sen, hep-th/9402032, Phys. Lett. 329B (1994) 217; C. Vafa and E. Witten, hep-th/9408074, Nucl. Phys. B432 (1994) 3.

[5] N. Seiberg and E. Witten, hep-th/9408099, Nucl. Phys. B431 (1994) 484.

[6] A. Hanany and Y. Oz, TAUP-2248-95, WIS-95/19, hep-th/9505075.

[7] P.C. Argyres, M.R. Plesser and A. Shapere, IASSNS-HEP-95/32, UK-HEP/9506, hep-th/9505100.

[8] N. Seiberg, hep-th/9411149, Nucl. Phys. B435 (1995) 129.

[9] E. Witten, Nucl. Phys. B202 (1982) 253

[10] N. Seiberg, hep-ph/9309335, Phys. Lett. 318B (1993) 469.

[11] D. Amati, K. Konishi, Y. Meurice, G.C. Rossi and G. Veneziano, Phys. Rep. 162 (1988) 169 and references therein.

[12] M.A. Shifman and A. I. Vainshtein, Nucl. Phys. B277 (1986) 456; Nucl. Phys. B359 (1991) 571

[13] J. Polchinski and N. Seiberg, (1988) unpublished.

[14] E. Witten, Nucl. Phys. B268 (1986) 79

[15] M. Dine and N. Seiberg, Phys. Rev. Lett. 57 (1986) 2625

[16] M.T. Grisaru, W. Siegel and M. Rocek, Nucl. Phys. B159 (1979) 429

[17] M.A. Shifman and A. I. Vainshtein, Nucl. Phys. B277 (1986) 456; Nucl. Phys. B359 (1991) 571.

[18] K. Intriligator, N. Seiberg, and S. Shenker, Phys. Lett. 342B (1995) 152.

[19] V. Kaplunovsky and J. Louis, Nucl. Phys. B422 (1994) 57.

[20] C.P. Burgess, J.-P. Derendinger, F. Quevedo, and M. Quiros, hep-th/9595171, CERN-Th/95-111.

[21] K. Intriligator, R.G. Leigh and N. Seiberg, hep-th/9403198, Phys. Rev. D50 (1994) 1092.

[22] K. Intriligator, hep-th/9407106, Phys. Lett. 336B (1994) 409.

[23] E. B. Bogomol'nyi, Sov. J. Nucl. Phys. 24 (1976) 449 
[24] M. K. Prasad and C. M. Somerfeld, Phys. Rev. Lett. 35 (1975) 760

[25] E. Witten and D. Olive, Phys. Lett. 78B (1978) 97

[26] D. Mumford and J. Fogarty, Geometric Invariant Theory (Springer, 1982).

[27] M. A. Luty and W. Taylor IV, hep-th/9506098, MIT-CTP-2440

[28] A.C. Davis, M. Dine and N. Seiberg, Phys. Lett. 125B (1983) 487

[29] I. Affleck, M. Dine and N. Seiberg, Nucl. Phys. B241 (1984) 493; Nucl. Phys. B256 (1985) 557.

[30] D. Finnell and P. Pouliot, RU-95-14, SLAC-PUB-95-6768, hep-th/9503115.

[31] V.A. Novikov, M.A. Shifman, A.I. Vainshtein and V.I. Zakharov, Nucl. Phys. B260 (1985) 157; A.I. Vainshtein, V.I. Zakharov, and M.A. Shifman, Sov. Phys. Usp. 28 (1985) 709.

[32] G. Veneziano and S. Yankielowicz Phys. Lett. 113B (1982) 231; T. Taylor, G. Veneziano and Yankielowicz, Nucl. Phys. B218 (1983) 439.

[33] N. Seiberg, hep-th/9402044, Phys. Rev. D49 (1994) 6857

[34] V. Novikov, M. Shifman, A. Vainshtein and V. Zakharov, Nucl. Phys. B229 (1983) 381

[35] T. Banks and A. Zaks, Nucl. Phys. B196 (1982) 189

[36] M. Flato and C. Fronsdal, Lett. Math. Phys. 8 (1984) 159; V.K. Dobrev and V.B. Petkova, Phys. Lett. 162B (1985) 127

[37] G. Mack, Comm. Math. Phys. 55 (1977) 1

[38] T.H.R. Skyrme, Proc.Roy.Soc. A260 (1961) 127; E. Witten, Nucl. Phys. B160 (1979) 57; Nucl. Phys. B223 (1983) 422; Nucl. Phys. B223 (1983) 433

[39] O. Aharony, hep-th/9502013, Phys. Lett. 351B (1995) 220.

[40] N. Seiberg and E. Witten, hep-th/9407087, Nucl. Phys. B426 (1994) 19.

[41] K. Intriligator and N. Seiberg, hep-th/9408155, Nucl. Phys. B431 (1994) 551.

[42] K. Intriligator and N. Seiberg, hep-th/9503179, Nucl. Phys. B444 (1995) 125.

[43] G. 'tHooft, Nucl. Phys. B190 (1981) 455.

[44] J. Cardy and E. Rabinovici, Nucl. Phys. B205 (1982) 1; J. Cardy, Nucl. Phys. B205 (1982) 17.

[45] K. Intriligator and N. Seiberg, hep-th/9506084, to appear in the Proc. of Strings 95

[46] P. Argyres and M. Douglas, hep-th/9505062, IASSNS-HEP-95-31.

[47] A.Yu. Morozov, M.A. Olshansetsky and M.A. Shifman, Nucl. Phys. B304 (1988) 291.

[48] K. Intriligator, N. Seiberg and S. Shenker, hep-ph/9410203, Phys. Lett. 342B (1995) 152.

[49] A. Klemm, W. Lerche, S. Theisen and S. Yankielowicz, hep-th/9411048, Phys. Lett. 344B (1995) 169; hep-th/9412158. 
[50] P. Argyres and A. Faraggi, hep-th/9411057, Phys. Rev. Lett. 74 (1995) 3931.

[51] D. Kutasov, hep-th/9503086, Phys. Lett. 351B (1995) 230.

[52] R. Leigh and M. Strassler, hep-th/9503121, Nucl. Phys. B447 (95) 1995

[53] U. Danielsson and B. Sundborg, USITP-95-06, UUITP-4/95, hep-th/9504102.

[54] M.R. Douglas and S.H. Shenker, RU-95-12, RU-95-12, hep-th/9503163.

[55] S. Elitzur, A Forge, A. Giveon and E. Rabinovici, RI-4-95 hep-th/9504080.

[56] O. Aharony, J. Sonnenschein and S. Yankielowicz, TAUP-2246-95, CERNTH/95-91, hep-th/9504113.

[57] D. Kutasov and A. Schwimmer, EFI-95-20, WIS/4/95, hep-th/9505004.

[58] K. Intriligator and P. Pouliot, hep-th/9505006, Phys. Lett. 353B (1995) 471.

[59] K. Intriligator, RU-95-27, hep-th/9505051, Nucl. Phys. B to appear.

[60] P.C. Argyres and M.R. Douglas, RU-95-31, hep-th/9505062.

[61] M. Berkooz, RU-95-29, hep-th/9505067.

[62] R. Leigh and M. Strassler, hep-th/9505088, RU-95-30.

[63] K. Intriligator, R. Leigh and M. Strassler, hep-th/9506148, RU-95-38.

[64] P. Pouliot, hep-th/9507018, RU-95-46

[65] I. Pesando, hep-th/9506139, NORDITA-95/42 P

[66] S.B. Giddings and J. M Pierre, hep-th/9506196, UCSBTH-95-14

[67] E. Poppitz and S. P. Trivedi, hep-th/9507169, EFI-95-44, Fermilab-Pub$95 / 258-\mathrm{T}$ 